\title{
Identification of Thermal Conductivity of Modern Materials Using the Finite Element Method and Nelder-Mead's Optimization Algorithm
}

\author{
Maria Nienartowicz and Tomasz Strek \\ Poznan University of Technology, \\ Institute of Applied Mechanics, \\ Poland
}

\section{Introduction}

Due to the fact that modern materials are widely used in aerospace industry, automotive industry, medicine and many others it is very important to find out a way to meet all its needful parameters. There is a lot of composites, which arise from a combination of at least two different components on the macroscopic level and whose parameters are unknown. Therefore, it is difficult to get to know their possibilities and functionality. A kind of composite materials are Functionally Graded Materials (FGM). They are characterized by the fact that its composition and structure gradually change over the volume, which follows from changes in properties of material (Miyamoto et al., 1999). There are many works on this topic.

Microstructure and thermal stress relaxation of $\mathrm{ZrO} 2-\mathrm{Ni}$, which is an example of FGM, by hot-pressing was studied in (Jingchuan et al., 1996). The researches consisted of scanning, transmission electron microscopy and X-ray diffractometry shown that the chemical composition and microstructure of $\mathrm{ZrO} 2-\mathrm{Ni}$ FGM is distributed gradiently in stepwise way. The preliminary analyze of thermal stress distribution by means of Finite Element Method was also presented there. In other article the identification of the nonlinear thermalconductivity coefficient by gradient method was shown (Borukhov, 2005). Author puts his attention to the gradient methods of solution of the inverse heat-conduction problem of designation of the nonlinear coefficient $\lambda(\mathrm{T})$, without preliminary finite-dimensional approximation. Review of the principal developments in Functionally Graded Materials, with an emphasis on the recent work published since 2000, are presented in (Birman\&Byrd, 2007). A various areas relevant to the different aspects of theory and applications of FGM are submitted there.

As it was mentioned above the difficulty of working with composites is that many parameters cannot be directly determined. An example would be the relationship between composite properties and temperature variation. The study of these relationships can be done experimentally, which is costly because it requires proper equipment and sample preparation. 
The effect of multi fibres filler in composite on thermal conductivity was examined in paper (Jopek\&Strek, 2011). Three types of optimization were performed in terms of effective thermal conductivity: minimization, maximization and determination of arrangement which gives expected value of effective thermal conductivity. Hybrid method combining optimization with genetic algorithm and differential equation solver by finite element method was used to find optimal arrangement of fibers position in composite matrix.

In this paper an attempt to perform simulation for comparison numerical results with experimental results was made. To carry out the simulation, the environment of the program COMSOL Multiphysics was used (Comsol, 2007). The code was written in COMSOL Script and combines Finite Element Method (Zienkiewicz\&Taylor, 2000) with optimization Nelder-Mead algorithm (Nelder\&Mead, 1965). This algorithm belongs to nongradient optimization methods of function of many variables (Weise, 2009). The computations are performed using the simplex, so that in each iteration value at several specific points is calculated. In this way we find the minimum of the function. It found its application in many studies and wide range of problems.

This method was used for engineering optimization as a Globalized Bounded Nelder-Mead algorithm (GBNM) (Luersen\&Le Riche, 2004). In that article a global approach to real optimization was shown by using restart procedure. The Globalized Bounded Nelder-Mead can be applied to discontinuous, non-convex functions. To speed up a global search an improved restart procedure was found. An example of this was shown in article where improved restart procedure was used for optimization of composite bracket (Hossein Ghiasi et al., 2007). This approach was changed by using a one-dimensional adaptive probability function and including nonlinear constraints. Thereby the Improved Globalized NelderMead Method became more efficient than evolutionary algorithm, as results confirmed. Some attempts of benchmarking the Nelder-Mead downhill simplex algorithm with many local restarts appeared in (Hansen, 2009). This method was also applied for Multiple Global Minima (Stefanescu, 2007). In that article author proves that Nelder-Mead heuristic procedure can detect successfully multiple global minima.

Simplex method, mentioned above, can be also used as combined with genetic algorithm. In this way the genetic algorithm is used to find a global optimum area and then the NelderMead algorithm is used for a local optimization (Durand\&Alliot, 1999). A hybrid genetic and Nelder-Mead algorithm (HGNMA) was also used for decoupling of Multiple Input Multiple Output (MIMO) system with application on two coupled distillation columns process (Lasheen et al., 2009). In that article a technique that uses relative gain array to choose proper pairing and HGNMA to find optimal elements' values of the steady state decoupling compensator unit was proposed. That minimizes internal couplings of MIMO systems. Similar hybrid was presented for optimization in the variational methods of Boundary Value Problems (Mastorakis, 2009). Author presents a way of solution of pLaplacian equation. Next, it is discussed with other methods for the solution. Using the Nelder-Mead's method also problems of identification of material parameters can be solved, as it is shown in article where investigation of processes in a rock mass is described (Blaheta et al., 2010).

Simulation, which is carried out in this chapter, for the heat transfer in considered domains with the boundary conditions in the form of a heat flux on both ends or temperature allows 
to determine parameters such as a thermal conductivity, a heat exchange surface area at the boundary or an outside temperature around both ends of the area. Modifying the program code simulations which optimize the temperature, in case when the thermal conductivity is dependent on the nonlinear function, can be also performed. The Nelder-Mead's optimization algorithm combined with Finite Element Method calculations are performed in COMSOL Script environment. Considered modern composite consists of multiple materials with different properties in different sections.

\section{Heat transfer}

The heat transfer can be defined as a movement of energy which is caused of temperature difference. It can be provided by the three mechanisms. First of them is a conduction, which can be described as diffusion, which is held in a stationary medium and occurs because of temperature gradient. The mentioned medium can be in form of solid or fluid. Next is convective, which appears as a result of fluid motion. The last one is radiation, which follows from electromagnetic waves between two surfaces, on which different temperatures are. Additionally those surfaces must comply with a condition that the first surface is visible to an infinitesimally small observer on the second surface.

The heat transfer by conduction can be defined by the heat equation

$$
\rho \cdot C_{p} \cdot \frac{\partial T}{\partial t}-\nabla \circ(k \cdot \nabla T)=Q,
$$

where: $\mathrm{T}$ - is the temperature, $\rho$ - is the density, $C_{p}$ - is the heat capacity at constant pressure, $\mathrm{k}$ - is the thermal conductivity and $\mathrm{Q}-$ is a heat source or heat sink. Taking into consideration a steady-state model the temperature does not change with time.

The thermal conductivity describes a relationship between the heat flux vector $\mathbf{q}$ and the gradient of temperature $\nabla T$ (Bejan\&Kraus, 2003), so it takes a form of

$$
\mathbf{q}=-\mathrm{k} \cdot \nabla \mathrm{T},
$$

The heat flux, mentioned above, is a kind of boundary condition, which can be described as

$$
\mathbf{n} \circ(\mathrm{k} \cdot \nabla \mathrm{T})=\mathbf{q}_{0}+\mathrm{h} \cdot\left(\mathrm{T}_{\mathrm{inf}}-\mathrm{T}\right)+\mathrm{C}_{\text {const }} \cdot\left(\mathrm{T}_{\mathrm{amp}}^{4}-\mathrm{T}^{4}\right),
$$

where: $\mathbf{q}_{0}$ - is the input heat flux, $h \cdot\left(\mathrm{T}_{\mathrm{inf}}-\mathrm{T}\right)$ - is used for convective heat transfer, where $\mathrm{h}$ is the heat transfer coefficient and $\mathrm{T}_{\mathrm{inf}}$ is the ambient bulk temperature, $\mathrm{C}_{\text {const }} \cdot\left(\mathrm{T}_{\mathrm{amp}}^{4}-\mathrm{T}^{4}\right)$ - is used for radiation heat transfer, where $\mathrm{T}_{\mathrm{amp}}$ is the temperature of surrounding radiation environment and $\mathrm{C}_{\text {const }}$ is a product of surface emissivity and the Stefan-Boltzmann constant.

\section{The Nelder-Mead algorithm}

The Nelder-Mead algorithm is a method that does not require to determine a derivative of an objective function. This function is determined in few specific points, different in each iteration. The first simplex algorithm was defined by Spendley in 1962. In 1965 Nelder and Mead improved it and turned the simplex search into an optimization algorithm by adding options like: reflection, expansion, contraction and shrinking. Thanks those operations, 
which speed up the process of optimization, the simplex deforms in way that was suggested by Nelder and Mead to adapt better to the objective functions (Nelder \& Mead, 1965).

An $\mathrm{n}$-dimensional simplex with $\mathrm{n}+1$ vertices $\mathrm{p} 0, \mathrm{p} 1, \mathrm{p} 2, \ldots, \mathrm{pn}$ is the smallest convex set which contains these points, where set $\{\mathrm{pj}-\mathrm{p} 0: 1 \leq \mathrm{j} \leq \mathrm{n}\}$ must consist of linearly independent vector. In the two-dimensional space the simplex can be created from any triangle and in three-dimensional space from any tetrahedra.

In this method selected initial simplex is modified by means of elementary geometric operations called: reflection, expansion, contraction and shrinking. As a result of each of them the vertex, where value of the objective function takes the highest value (the "worst" vertex), is replaced by another - "better". In this way the simplex is coming more and more to local minimum of examined function.

Finding the minimum of the objective function must be preceded by an analysis, where as a result the vertices, in which the objective function takes the smallest and the highest value, are marked in the following way (see Fig. 1a):

- $\quad$ pmin - the vertex where the objective function takes the smallest value:

$$
\mathrm{f}(\mathrm{pmin}) \leq \mathrm{f} \text { (pi) for any } 0 \leq \mathrm{i} \leq \mathrm{n}
$$

- $\quad$ max - the vertex where the objective function takes the highest value:

$$
\mathrm{f}(\mathrm{pmax}) \geq \mathrm{f} \text { (pi) for any } 0 \leq \mathrm{i} \leq \mathrm{n}
$$

- $\quad \bar{p}$ - centroid of the points (the vertex pmax is excluded) see:

$$
\overline{\mathrm{p}}=\frac{1}{\mathrm{n}} \cdot\left(\sum_{\mathrm{i} \neq \max } p i\right)
$$

After determining points pmin, $\overline{\mathrm{p}}$, pmax, a procedure of minimization of the objective function can begin. In each iteration the following stages can be specified: reflection, expansion, contraction and shrinking, which are described below (Weise, 2009).

Reflection - is based on determining a point which is symmetrical image of point pmax relative to point $\bar{p}$. New point is marked as podb (see Fig. 1b) and its coordinates are designed by formula:

$$
\operatorname{pod} b=\bar{p}+a \cdot(\bar{p}-p m a x)
$$
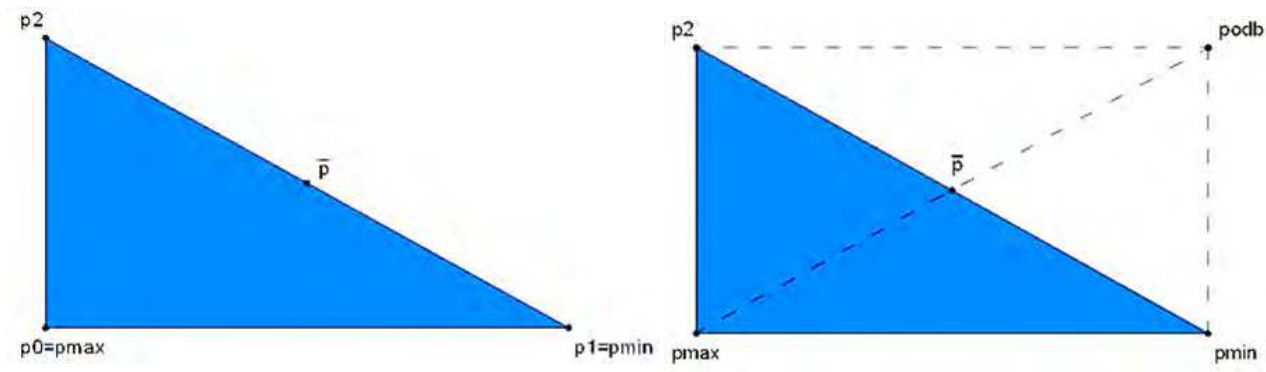

Fig. 1. (a) Initial simplex with vertices $p 0, p 1$, p2 where $(f(p 0)>f(p 2)>f(p 1)),(b)$ Simplex: reflection stage 
Value of reflection coefficient $\alpha$ is in the range of $\alpha \in(0,1]$, but usually it is assumed that $\alpha=1$.

After reflection stage, depending on value of the objective function in reflection point $\mathrm{f}($ podb), we consider few excluded cases (8), (9) and (10), which determine further investigation in given iterations:

$$
\begin{gathered}
\mathrm{f}(\text { pmin }) \leq \mathrm{f}(\text { podb })<\mathrm{f}(\text { pmax }), \\
\mathrm{f}(\text { podb })<\mathrm{f}(\text { pmin }), \\
\mathrm{f}(\max ) \leq \mathrm{f}(\text { podb }),
\end{gathered}
$$

If in calculated point podb the objective function takes value (8) then the reflection is accepted. The new simplex is designed by replacing the vertex pmax with podb. Next indexes min, max and location of point $\overline{\mathrm{p}}$ are updated and if a stop condition, which is described later, is not fulfilled a new iteration begins with new reflection.

Expansion - Assume that reflection inequality (9) was fulfilled, which means that a vertex which was found in reflection stage is better point than pmin (it is closer to minimum of objective function $\mathrm{f}$ ).

It suggests that next steps of finding the minimum should follow in this direction. Because of this the reflection is not accepted and the calculations are carried out by the expansion (see Fig. 2). A new point is calculated and marked as pe:

$$
\mathrm{pe}=\overline{\mathrm{p}}+\gamma \cdot(\operatorname{podb}-\overline{\mathrm{p}}),
$$

where $\gamma>1$ is an expansion coefficient (usually $\gamma=2$ ). Next, a value of the objective function in new point is calculated $f(p e)$, and:

- if $\mathrm{f}($ podb $)<\mathrm{f}($ pmin) then the expansion is successful, new simplex is designed by replacing pmax with pe (new simplex is designed by vertices pe, p2, pmin - Fig. 2a); then indexes min and max and a location of point $\bar{p}$ are updated and after checking the stop condition next iteration begins;

- else when $f(p e) \geq f($ podb), pmax is replaced by podb (new simplex is designed by vertices podb, p2, pmin - Fig. 2b) and it follows as previous (indexes are updated, stop condition is checked and next iteration begins)
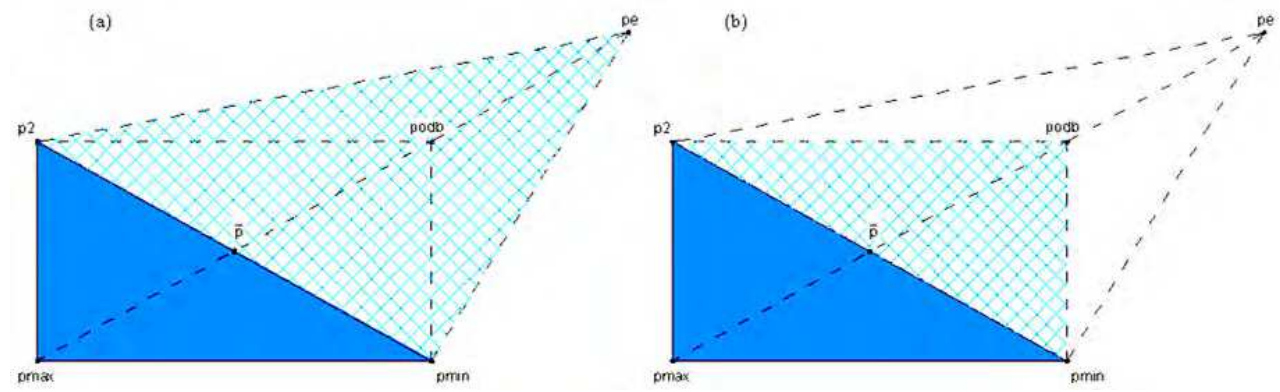

Fig. 2. Expansion stages: successful (a), unsuccessful (b) (new designed simplex is hatched) 
Contraction. Reflection cannot be accepted also in case when $f(o d b) \geq f(p m a x)$, see (10). In this situation occurs contraction of a simplex, whose new vertex is counted according to the formula:

$$
\mathrm{pz}=\overline{\mathrm{p}}+\beta \cdot(\operatorname{pmax}-\overline{\mathrm{p}})
$$

where a coefficient of contraction $\beta$ takes a value $\beta \in(0,1)$, usually $\beta=0.5$ (see Fig. 3a). If point $\mathrm{pz}$ leads to improvement, which means $\mathrm{f}(\mathrm{pz})<\mathrm{f}(\mathrm{pmax})$, then point pmax is replaced by point $\mathrm{pz}$ and a new simplex is created (designated by pz, p2, pmin). Next indexes are updated, stop condition is checked and next iteration begins.

Shrinking. This stage takes place when after contraction an inequality (13) is fulfilled:

$$
f(p z) \geq f(p m a x)
$$

In this situation point pmin remains unchanged, and the whole simplex is shrinking according formula (14):

$$
\mathrm{pi} \leftarrow \delta \cdot(\mathrm{pi}+\mathrm{pmin}), \mathrm{i}=0,1, \ldots, \mathrm{n}, \mathrm{i} \neq \min (14)
$$

where $\delta \in(0,1)$ is a shrinking coefficient and usually $\delta=0,5$ (see Fig. 3b). A simplex which is build of a new obtained points $\mathrm{p} 0, \ldots, \mathrm{pn}$ is used in next iteration (if the stop condition is not fulfilled).
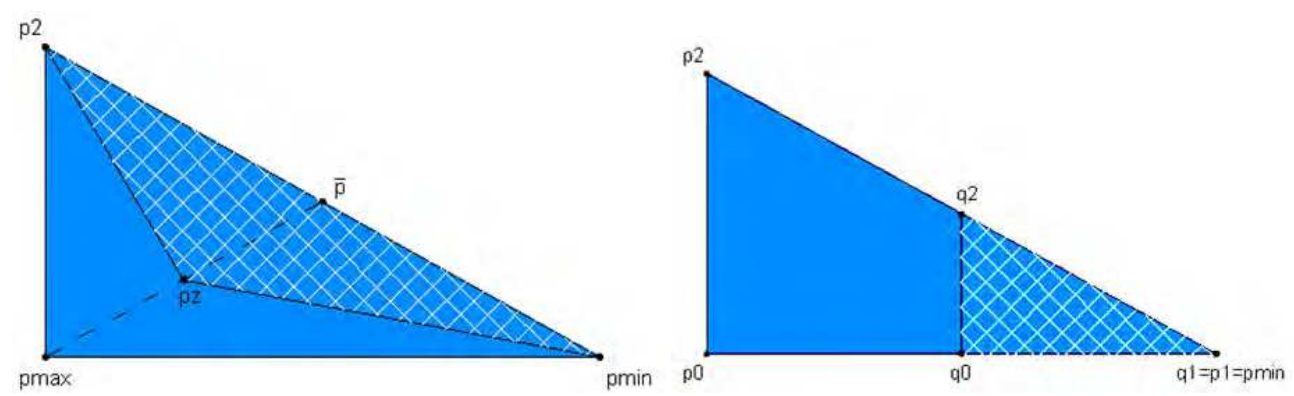

Fig. 3. (a) Contraction stage (if contraction is successful the hatched simplex is chosen), (b) Shrinking stage (new designed simplex is hatched)

In this papers two stop conditions were used. The first when an absolute value of difference between $f(p m i n)$ and $f(p m a x)$ is smaller than accuracy solution

$$
\operatorname{abs}(\mathrm{f}(\text { pmin })-f(p m a x))<\varepsilon
$$

and the second when a number of iterations is bigger than maximum number of iterations

$$
\text { step }>\text { maxstep. }
$$

Algorithm of Nelder-Mead method

Input data:

Initial simplex with vertices: p0, p1, .., pn, 
Coefficients:

a - reflection,

$\beta$ - contraction,

$\gamma$ - expansion,

$\delta$ - shrinking,

$\varepsilon$ - accuracy of solution,

maxstep - maximum number of iterations.

1. Repeat

2. count a value of function in vertices of simplex: $p 0, p 1, \ldots, p n$

3. find pmin, $\operatorname{pmax}(\min \neq \max )$

4. $\overline{\mathrm{p}}=\frac{1}{\mathrm{n}} \cdot\left(\sum_{i \neq \max } \mathrm{pi}\right)$,

5. $\operatorname{podb}=\overline{\mathrm{p}}+\mathrm{a} \cdot(\overline{\mathrm{p}}-\mathrm{pmax})$

6. if $\mathrm{f}($ podb $)<\mathrm{f}(\mathrm{pmin})$ then

7. $\mathrm{pe}=\overline{\mathrm{p}}+\gamma \cdot($ podb $-\overline{\mathrm{p}})$

8. if $\mathrm{f}(\mathrm{pe})<\mathrm{f}($ podb $)$ then

9. $\quad \mathrm{pmax}=\mathrm{pe}$

10. else

11. $\quad \operatorname{pmax}=\mathrm{podb}$

- expansion

12. end if

13. else

14. if $\mathrm{f}($ pmin $) \leq \mathrm{f}($ podb $)<\mathrm{f}($ pmax $)$ then

15. $\quad$ max $=$ podb

16. else

17. $\quad \mathrm{pz}=\overline{\mathrm{p}}+\beta \cdot(\operatorname{pmax}-\overline{\mathrm{p}})$

18. if $f(p z) \geq f(p m a x)$ then

19. for $\mathrm{i}=0$ to $\mathrm{n}$ do

20. if $\mathrm{i} \neq \mathrm{min}$ then

21. $\mathrm{pi}=\delta \cdot(\mathrm{pi}+\mathrm{pmin}) \longrightarrow$ shrinking

22. end if

23. end for

24. else

25. $\quad \operatorname{pmax}=\mathrm{pz}$

26. end if

27. end if

28. end if

29. until abs $(\mathrm{f}($ pmin $)-\mathrm{f}($ pmax $))<\varepsilon$ or step $>$ maxstep

stop conditions

30. return $x^{*}=$ pmin

approximate solution

\section{Reconstruction of thermal parameters in 1-D domain}

As a first a reconstruction of thermal parameters was carried out. This simulation was made for heat transfer in 1-D space, in a domain which length was $1 \mathrm{~m}$. The boundary condition was the heat flux at both ends of the domain. Basing on the temperature distribution in area $T(x)$ thermal parameters of the issue were designated. Those parameters were: a thermal 
conductivity $(k)$, a transversal convective heat transfer coefficient ( $h 1$ and $h 2)$ and an external temperature around both ends of the domain (Tinf1 and Tinf2). The reconstruction of mentioned factors was performed by the optimization.

\subsection{Stage I}

In the first stage desired temperature distribution was defined as

$$
\overline{\mathrm{T}}(\mathrm{x})=18.75 \cdot \mathrm{x}+287.5,
$$

and minimized integral have a form of

$$
F=\int_{0}^{1} \operatorname{Abs}(\mathrm{T}(\mathrm{x})-\overline{\mathrm{T}}(\mathrm{x})) \mathrm{d} \mathrm{x}=\int_{0}^{1} \operatorname{Abs}(\mathrm{T}(\mathrm{x})-(18.75 \cdot \mathrm{x}+287.5)) \mathrm{d} \mathrm{x} .
$$

The start simplex for particular parameters is presented in Table 1.

\begin{tabular}{|c|c|c|c|c|c|}
\hline Vertices & $\sqrt{k}$ & $\sqrt{h 1}$ & $\sqrt{h 2}$ & $\sqrt{\text { Tinf } 1}$ & $\sqrt{\text { Tinf2 }}$ \\
\hline $\mathrm{p}_{1}$ & 1 & 2 & 3 & 4 & 5 \\
\hline $\mathrm{p}_{2}$ & 100 & 2 & 3 & 4 & 5 \\
\hline $\mathrm{p}_{3}$ & 1 & 100 & 3 & 4 & 5 \\
\hline $\mathrm{p}_{4}$ & 1 & 2 & 100 & 4 & 5 \\
\hline $\mathrm{p}_{5}$ & 1 & 2 & 3 & 100 & 5 \\
\hline $\mathrm{p}_{6}$ & 1 & 2 & 3 & 4 & 100 \\
\hline
\end{tabular}

Table 1. Start simplex for the stage I

Numbers, placed in Table 1, are square roots of searched thermal parameters. This assumption guarantees that values will be positive. Results of the calculations are presented below in Table 2 and Table 3. Required accuracy of solution was obtained after 88 steps with $\mathrm{F}=0.0105$ for following set of parameters.

\begin{tabular}{|c|c|c|c|c|c|}
\hline Parameter & $\sqrt{k}$ & $\sqrt{h 1}$ & $\sqrt{h 2}$ & $\sqrt{\operatorname{Tinf1}}$ & $\sqrt{\operatorname{Tinf2}}$ \\
\hline $\mathrm{p}_{\min }$ & 53.1230 & 16.4196 & 42.9727 & 9.5704 & -18.2983 \\
\hline
\end{tabular}

Table 2. Values of minimized square roots of pmin

\begin{tabular}{|c|c|c|c|c|c|}
\hline Parameter & $k$ & $h 1$ & $h 2$ & Tinf1 & Tinf2 \\
\hline $\mathrm{p}^{2}$ min & 2800 & 269.6035 & 1800 & 91.5928 & 334.8276 \\
\hline
\end{tabular}

Table 3. Values of minimized parameters pmin

The objective function $F$ was minimized with the accuracy of solution $\varepsilon=1 \mathrm{e}-2$. 


\subsection{Stage II}

In the second stage of calculation some restrictions were imposed to the optimal parameters, as it is presented below:

$$
\begin{gathered}
80<\mathrm{k}<120, \\
8<\mathrm{h} 1<12, \\
15<\mathrm{h} 2<25, \\
80<\operatorname{Tinf} 1<120, \\
350<\operatorname{Tinf} 2<45 .
\end{gathered}
$$

Thereby, we did not have to minimize the roots of objective function.

The start simplex for particular parameters is presented in Table 4.

\begin{tabular}{|c|c|c|c|c|}
\hline$k$ & $h 1$ & $h 2$ & Tinf1 & Tinf2 \\
\hline 80 & 8 & 15 & 80 & 350 \\
\hline 120 & 12 & 25 & 120 & 450 \\
\hline 82 & 9 & 18 & 90 & 360 \\
\hline 110 & 11 & 24 & 119 & 420 \\
\hline 101 & 8 & 17 & 89 & 360 \\
\hline 110 & 11 & 21 & 111 & 444 \\
\hline
\end{tabular}

Table 4. Start simplex for the stage II

Numbers placed in Table 4 are not square roots of searched thermal parameters, but there are values which minimize the objective function.

Required accuracy of solution was obtained after 45 steps with $F=0.1801$ for following set of parameters.

\begin{tabular}{|c|c|c|c|c|c|}
\hline Parameter & $k$ & $h 1$ & $h 2$ & Tinf1 & Tinf2 \\
\hline$p_{\text {min }}$ & 101.0347 & 9.8034 & 20.0654 & 101.9420 & 396.8020 \\
\hline
\end{tabular}

Table 5. Values of minimized parameters pmin

Values which were determined are within established limits.

\subsection{Stage III}

Next stage of the research was optimization of the thermal parameters of material in which coefficient of thermal conductivity was dependent on spatial variable $x$, like in the Functionally Graded Materials. In those composites temperature distribution with given 
boundary conditions are usually nonlinear. In these paper it is assumed that parameter $k(x)$ has polynomial form

$$
\mathrm{k}(\mathrm{x})=p_{0}+p_{1} \mathrm{x}+p_{2} x^{2} .
$$

In this stage the heat transfer equation takes a form of

$$
\nabla \circ(k(x) \cdot \nabla T)=0,
$$

where $\mathrm{k}(\mathrm{x})$ - thermal conductivity depends on spatial variable $x$.

The following boundary conditions (different temperature on ends) was assumed for calculations

$$
\begin{aligned}
& \mathrm{T}=\mathrm{T}_{01}=283 \mathrm{~K}, \\
& \mathrm{~T}=\mathrm{T}_{02}=483 \mathrm{~K} .
\end{aligned}
$$

Now the vector of parameters have a form of: $p=\left[p_{0}, p_{1}, p_{2}\right]$.

There were two tasks solved for one function of $\overline{\mathrm{T}}(\mathrm{x})$, one integral and for two different start simplexes.

The desired function $\overline{\mathrm{T}}(\mathrm{x})$ has a form of:

$$
\overline{\mathrm{T}}_{1}(\mathrm{x})=286.25-170.513 \cdot \mathrm{x}+2398.87 \cdot x^{2}-3898.57 \cdot x^{3}+2133.67 \cdot x^{4}-265 \cdot x^{5} .
$$

Minimized integral was defined as:

$$
\mathrm{I}_{1}=\int_{0}^{1} \operatorname{Abs}\left(\mathrm{T}(\mathrm{x})-\left(286.25-170.513 \cdot \mathrm{x}+2398.87 \cdot x^{2}-3898.57 \cdot x^{3}+2133.67 \cdot x^{4}-265 \cdot x^{5}\right)\right) \mathrm{dx}
$$

\subsubsection{Task 1}

The calculation began with the start simplex described in Table 6.

\begin{tabular}{|c|c|c|c|}
\hline Vertices, $p_{i j}$ & $p_{i 0}$ & $p_{i 1}$ & $p_{i 2}$ \\
\hline$p_{1 j}$ & 10 & -150 & 100 \\
\hline$p_{2 j}$ & 30 & -50 & 200 \\
\hline$p_{3 j}$ & 15 & -120 & 120 \\
\hline$p_{4 j}$ & 25 & -80 & 180 \\
\hline
\end{tabular}

Table 6. Start simplex for the task 1 of stage III

Results were achieved with solution accuracy of 1e-6.

Required accuracy of solution was obtained after 61 steps with $F=2.9492$ for following set of parameters.

\begin{tabular}{|c|c|c|c|}
\hline Parameter & $p_{0}$ & $p_{1}$ & $p_{2}$ \\
\hline$p_{\min }$ & 21.1025 & -106.6785 & 167.7030 \\
\hline
\end{tabular}

Table 7. Values of minimized parameters pmin 
In Fig. 4 disparity between the expected and the obtained temperature distribution is presented. Distribution of the coefficient of thermal conductivity, for $\mathrm{k}\left(\mathrm{x}, \mathrm{p}_{\min }\right)$ is presented in Fig. 5.

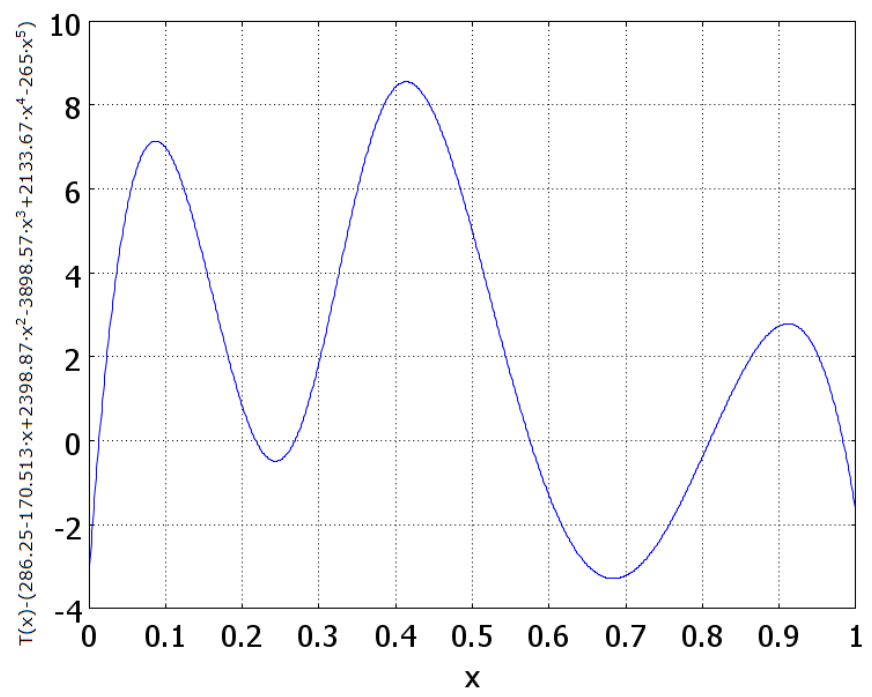

Fig. 4. Disparity between the expected and the obtained temperature distribution (task 1$)$

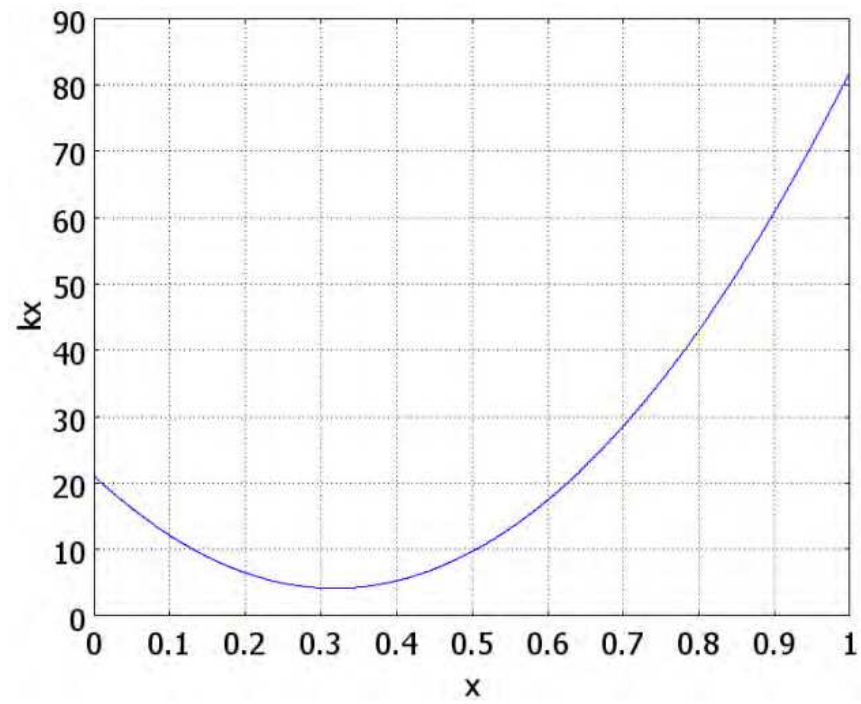

Fig. 5. Distribution of the thermal conductivity coefficient for $k\left(x, p_{\min }\right)(\operatorname{task} 1)$ 


\subsubsection{Task 2}

In the second task the start simplex was (Table 8):

\begin{tabular}{|c|c|c|c|}
\hline Vertices, $p_{i j}$ & $p_{i 0}$ & $p_{i 1}$ & $p_{i 2}$ \\
\hline$p_{1 j}$ & 19 & -115 & 130 \\
\hline$p_{2 j}$ & 16 & -95 & 160 \\
\hline$p_{3 j}$ & 18 & -110 & 150 \\
\hline$p_{4 j}$ & 21 & -90 & 170 \\
\hline
\end{tabular}

Table 8. Start simplex for the stage III (task 2)

Results were achieved with solution accuracy of 1e-6.

Required accuracy of solution was obtained after 151 steps with $F=2.9336$ for the following set of parameters.

\begin{tabular}{|c|c|c|c|}
\hline Parameter & $p_{0}$ & $p_{1}$ & $p_{2}$ \\
\hline$p_{\min }$ & 22.9741 & -114.6551 & 180 \\
\hline
\end{tabular}

Table 9. Values of minimized parameters pmin

In Fig. 6 disparity between the expected and the obtained temperature distribution is presented. Distribution of the coefficient of thermal conductivity, for $\mathrm{k}\left(\mathrm{x}, \mathrm{p}_{\min }\right)$ is presented in Fig. 7.

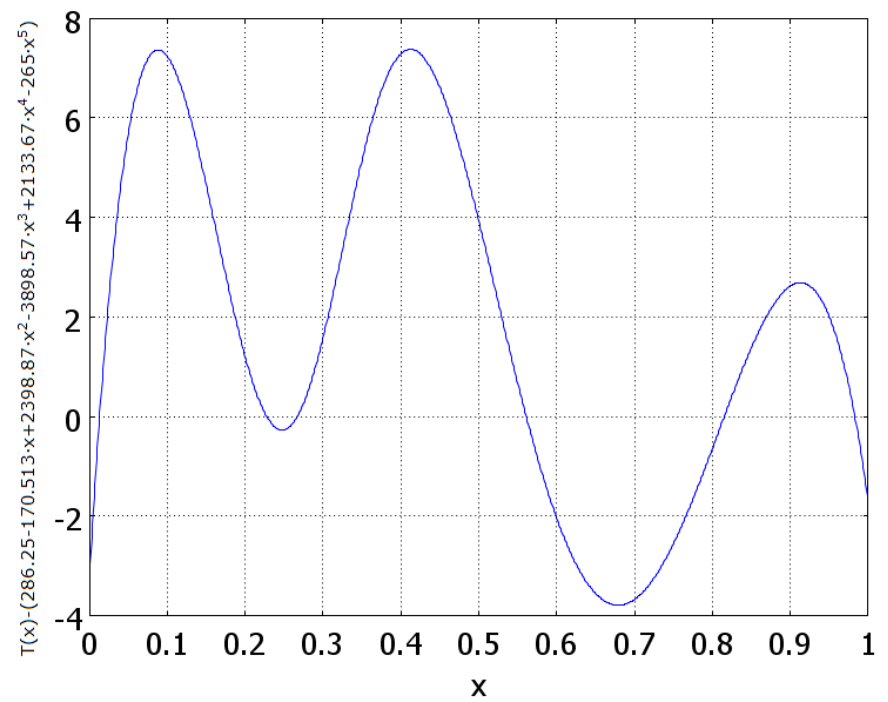

Fig. 6. Disparity between the expected and the obtained temperature distribution (task 2) 


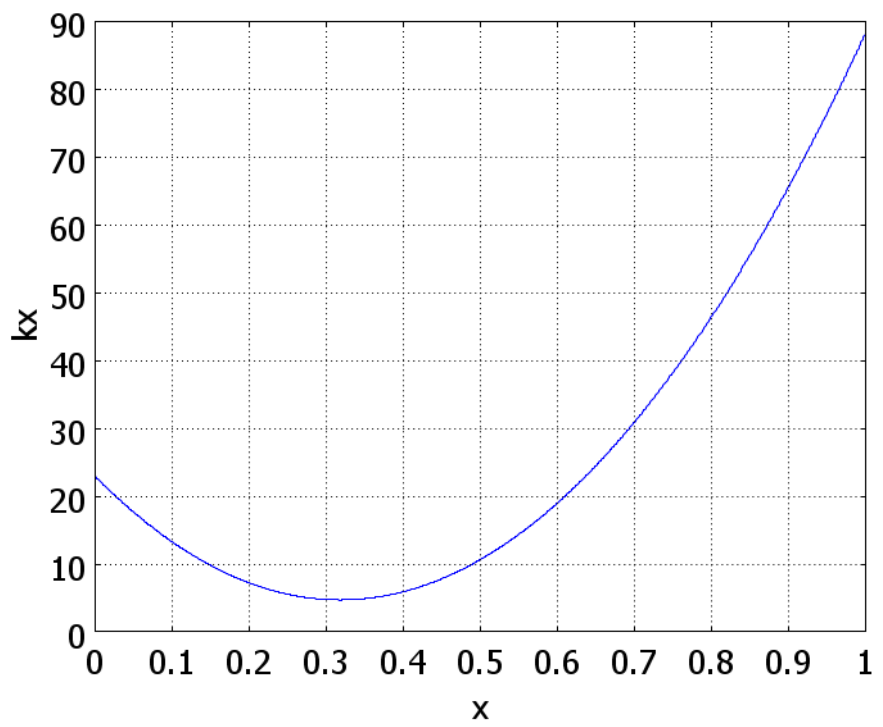

Fig. 7. Distribution of the thermal conductivity coefficient for $k\left(x, p_{\min }\right)(\operatorname{task} 2)$

Despite the fact that the thermal conductivity coefficient seems to look identical in task 1 and 2, there is some difference. In Fig. 8 the disparity in distribution of mentioned thermal conductivity is shown. Concluding, although in task 2 the start simplex was wider (bigger) than in task 1, the solution was found with the same accuracy.

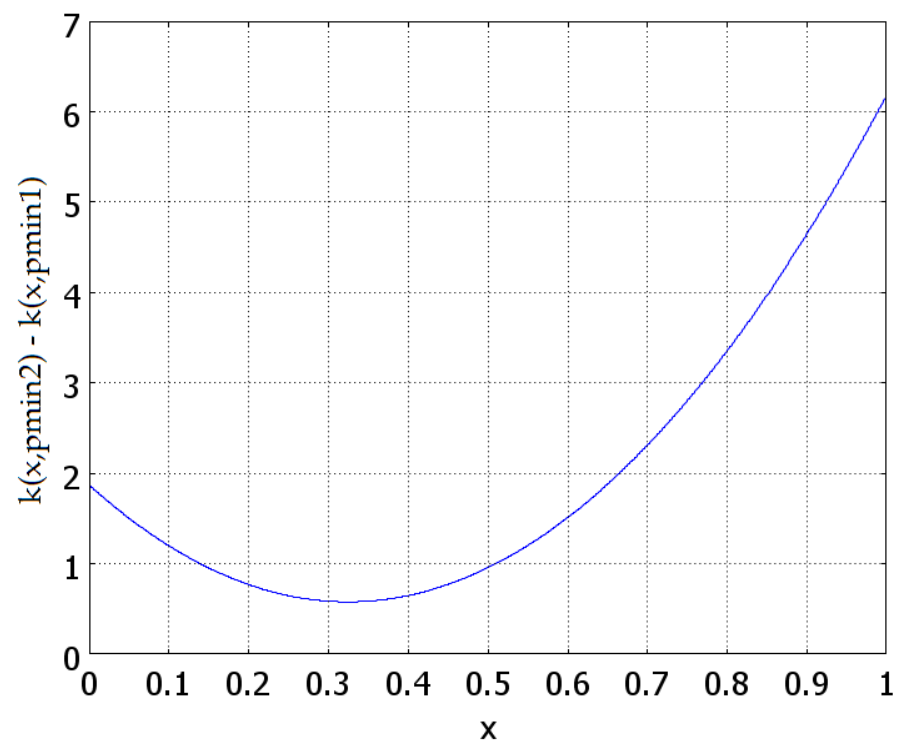

Fig. 8. Disparity between the thermal conductivity coefficient in task 2 and task 1 
Summarizing, as it was shown above in this section, it is possible to provide a reconstruction of parameters using hybrid method (FEM with Neleder-Mead). Carrying out the simulation for 1-D domain with length $1 \mathrm{~m}$ and defined boundary condition the parameters (the thermal conductivity, the transversal convective heat transfer coefficient and the external temperature around both ends of the domain) can be designated. Values of those parameters can be calculated within some restrictions, which can be specified for material which is examined. It is also possible to designate the value of thermal conductivity parameter of FGM which has a polynomial form.

\section{Reconstruction of thermal parameters in 2-D space}

In this subsection calculations were made to designate the distribution of the thermal conductivity in 2-D domain. Cylinder with radius $\mathrm{r}=1 \mathrm{~m}$ and height $\mathrm{z}=1 \mathrm{~m}$ was analysed in $2 \mathrm{D}$ axial symmetry model. An axis of symmetry is designated as $\mathrm{r}=0$. In this case it was also assumed that the distribution of the thermal conductivity has a form of polynomial as:

$$
\mathrm{k}(\mathrm{z})=p_{0}+p_{1} \cdot \mathrm{z}+p_{2} \cdot \mathrm{z}^{2}+p_{3} \cdot \mathrm{z}^{3} .
$$

A boundary condition, such that temperature at the top and at the bottom of the cylinder was equal in order that $T_{01}=400 \mathrm{~K}$ and $T_{02}=300 \mathrm{~K}$. For axis $\mathrm{r}=0$ axial symmetry was assumed and on the circumference of the cylinder was assumed a thermal insulation.

In these calculations some restrictions have been imposed. An integral I, which contains sum of three integrals was minimized, as shown below

$$
\mathrm{I}=\mathrm{I} 1+5 \cdot(1-\mathrm{I} 2)+5 \cdot(1-\mathrm{I} 3)
$$

where: I is minimized integral, I1 - is an absolute value of difference between expected and obtained temperature distribution

$$
\mathrm{I} 1=\int_{0}^{1} \operatorname{Abs}(\mathrm{T}(\mathrm{z})-\overline{\mathrm{T}}(\mathrm{z})) \mathrm{dz},
$$

I2 - determined part of domain where a relationship such that $k(z)>k m i n$, where $k m i n$ is a minimum value, is satisfied,

I3 - determined part of domain where a relationship such that $k(z)<k$ max, where $k$ max is a maximum value, is satisfied.

There were two tasks computed, each for one function of expected temperature. Each task was calculated for two variants of values for kmin and kmax - each of them for three start simplexes, as it is presented in subsection below.

\subsection{Task 1 - First function of temperature distribution}

In this task the expected temperature distribution took a form of

$$
\overline{\mathrm{T}}_{1}(\mathrm{z})=300.481+171.955 \cdot \mathrm{z}-72.9167 \cdot \mathrm{z}^{2},
$$

and integral I1 was defined as

$$
\mathrm{I} 1=\int_{0}^{1} \operatorname{Abs}\left(\mathrm{T}(\mathrm{z})-\left(300.481+171.955 \cdot \mathrm{z}-72.9167 \cdot \mathrm{z}^{2}\right)\right) \mathrm{dz} .
$$




\begin{tabular}{|c|c|c|c|c|c|}
\hline & Vertices, $p_{i j}$ & $p_{i 0}$ & $p_{i 1}$ & $p_{i 2}$ & $p_{i 3}$ \\
\hline \multirow{5}{*}{$\begin{array}{l}\text { Start simplex A } \\
\text { (the narrowest) }\end{array}$} & $p_{1 j}$ & 20 & 110 & -55 & 0 \\
\hline & $p_{2 j}$ & 30 & 120 & -50 & 1 \\
\hline & $p_{3 j}$ & 40 & 130 & -45 & 1 \\
\hline & $p_{4 j}$ & 50 & 140 & -40 & 0 \\
\hline & $p_{5 j}$ & 25 & 140 & -60 & 1 \\
\hline \multirow{5}{*}{$\begin{array}{l}\text { Start simplex B } \\
\quad \text { (wider) }\end{array}$} & $p_{1 j}$ & 50 & 200 & -65 & 5 \\
\hline & $p_{2 j}$ & -40 & -100 & -90 & 15 \\
\hline & $p_{3 j}$ & 45 & 180 & 5 & 20 \\
\hline & $p_{4 j}$ & 80 & 90 & -10 & 25 \\
\hline & $p_{5 j}$ & 70 & 180 & -120 & 30 \\
\hline \multirow{5}{*}{$\begin{array}{c}\text { Start simplex } C \\
\text { (the widest) }\end{array}$} & $p_{1 j}$ & 100 & 400 & -70 & 10 \\
\hline & $p_{2 j}$ & -50 & -200 & -130 & 20 \\
\hline & $p_{3 j}$ & 50 & 250 & 20 & 100 \\
\hline & $p_{4 j}$ & 110 & 20 & -150 & 250 \\
\hline & $p_{5 j}$ & 100 & 200 & -300 & 70 \\
\hline
\end{tabular}

Table 10. Task 1 - Different start simplexes taken for calculation

Three different start simplexes were assumed and collected in Table 10. As it was mentioned above there were two variants of calculations. Results and assumptions for them are presented in subsections 5.1.1 and 5.1.2.

\subsubsection{Variant 1}

For calculations below we defined restrictions as follows: $k m i n=20$ and $k m a x=120$. Which means that we were looking for $\mathrm{k}(\mathrm{z})$ distribution in this range: $20<\mathrm{k}(\mathrm{z})<120$. Numerical results are presented in table below.

\begin{tabular}{|c|c|c|c|c|c|c|}
\hline Simplex & Steps & $p_{i 0}$ & $p_{i 1}$ & $p_{i 2}$ & $p_{i 3}$ & Fmin \\
\hline A & 255 & $1.98993 \mathrm{e}+1$ & $2.71751 \mathrm{e}+1$ & $-3.02331 \mathrm{e}+1$ & $8.22635 \mathrm{e}+1$ & $1.54875 \mathrm{e}-1$ \\
\hline B & 208 & $2.11709 \mathrm{e}+1$ & $1.24838 \mathrm{e}+1$ & $1.17308 \mathrm{e}+1$ & $4.99511 \mathrm{e}+1$ & $3.02988 \mathrm{e}-1$ \\
\hline C & 121 & $1.99063 \mathrm{e}+1$ & $2.77594 \mathrm{e}+1$ & $-3.17098 \mathrm{e}+1$ & $8.33822 \mathrm{e}+1$ & $1.50866 \mathrm{e}-1$ \\
\hline
\end{tabular}

Table 11. Values of minimized parameters pmin for simplexes A, B, C

For the start simplex A, distribution of the thermal conductivity for minimized value $\mathrm{k}(\mathrm{pmin})$ is shown in Fig. 9. Disparity between the expected and the obtained temperature distribution was also examined (see Fig. 10) and it varies between -0.5 and 0.47. 


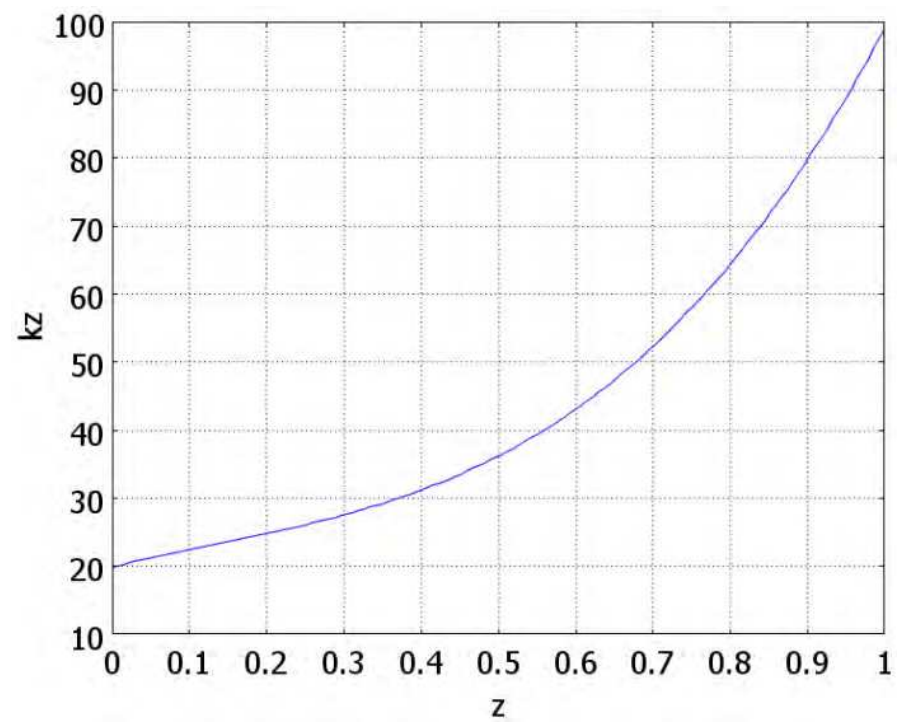

Fig. 9. Distribution of the thermal conductivity (variant 1, start simplex A)

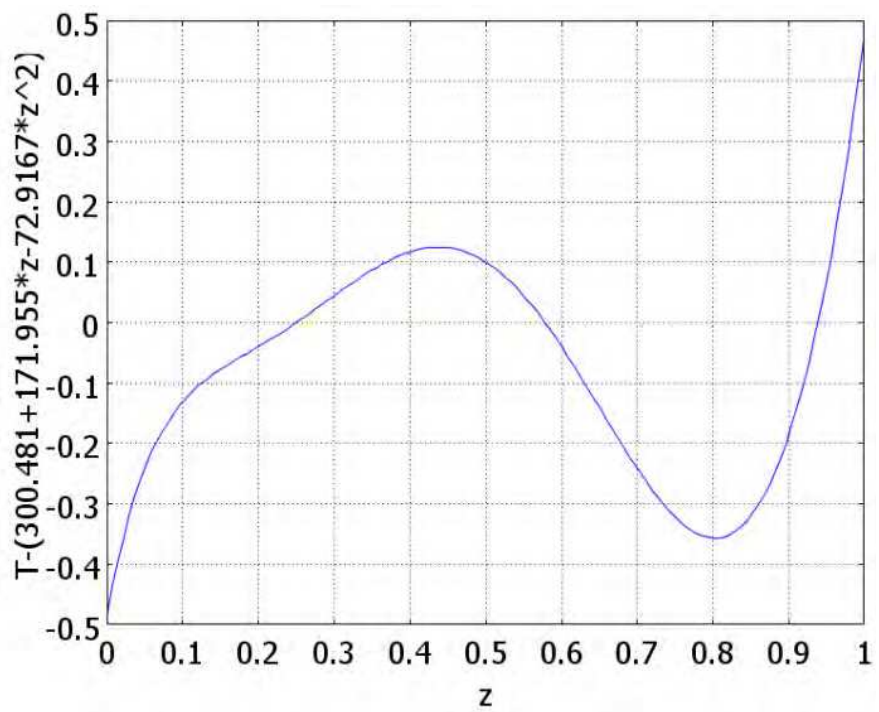

Fig. 10. Disparity between the expected and the obtained temperature distribution (variant 1, start simplex A)

For the start simplex $B$, distribution of the thermal conductivity for minimized value $\mathrm{k}$ (pmin) is shown in Fig. 11. Disparity between the expected and the obtained temperature distribution was also examined (see Fig. 12) and it varies between -0.6 and 0.45. 


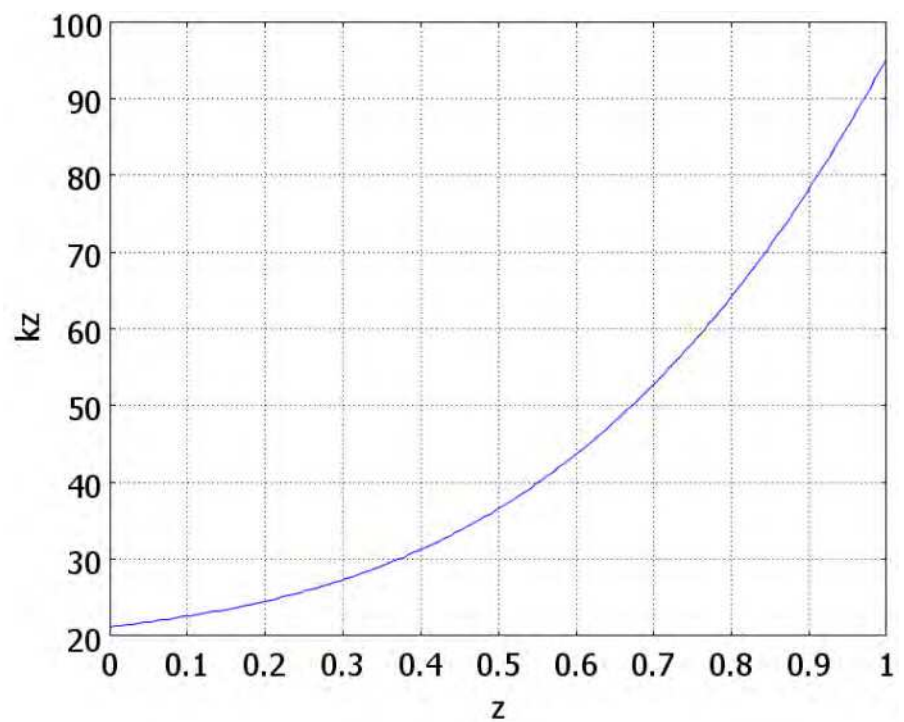

Fig. 11. Distribution of the thermal conductivity (variant 1, start simplex B)

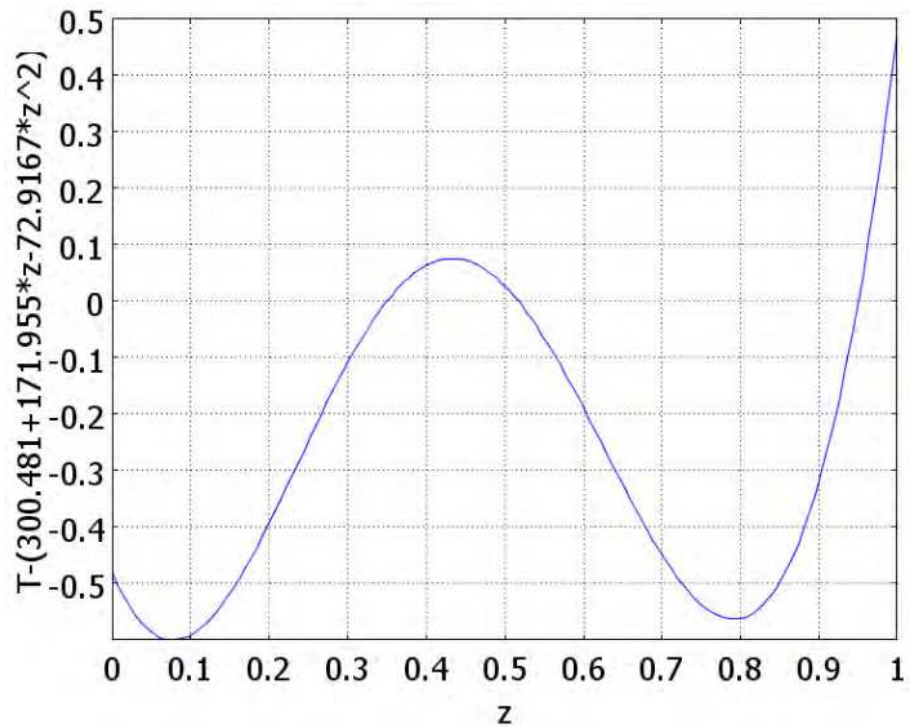

Fig. 12. Disparity between the expected and the obtained temperature distribution (variant 1 , start simplex B)

For the start simplex $C$, distribution of the thermal conductivity for minimized value $\mathrm{k}$ (pmin) is shown in Fig. 13. Disparity between the expected and the obtained temperature distribution was also examined (see Fig. 14) and it varies between -0.48 and 0.46 . 


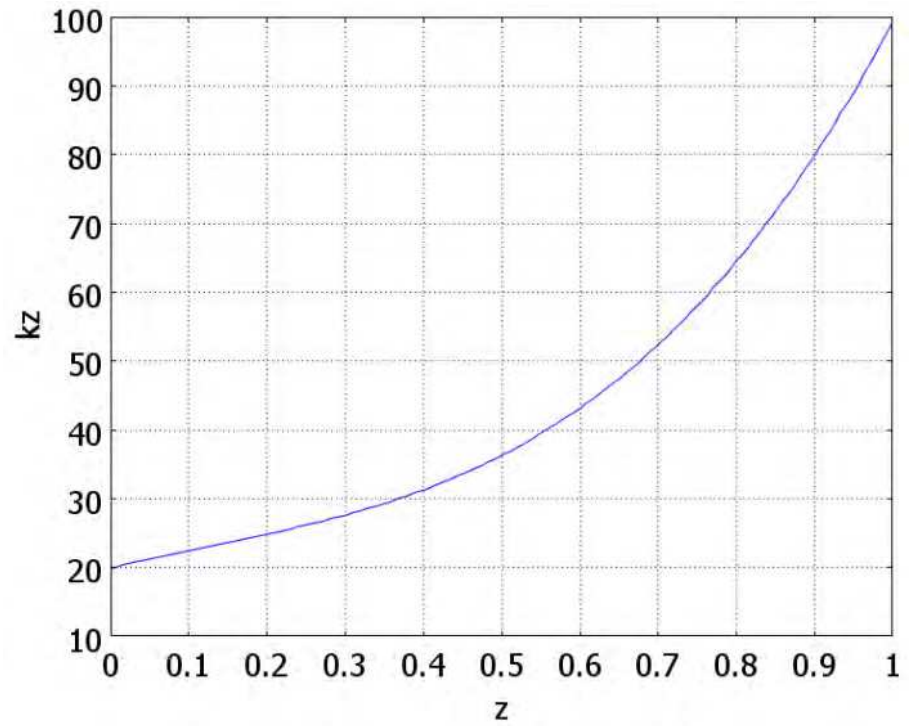

Fig. 13. Distribution of the thermal conductivity (variant 1 , start simplex C)

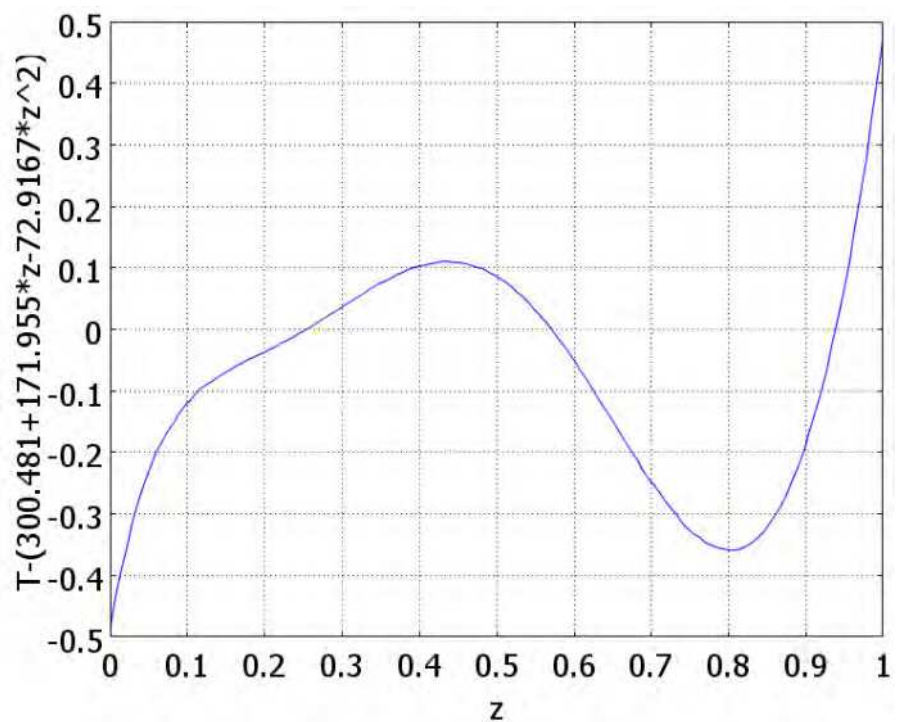

Fig. 14. Disparity between the expected and the obtained temperature distribution (variant 1 , start simplex C) 
In all cases the results were achieved with solution accuracy of 1e-5. Temperature distribution was similar for all simplexes.

\subsubsection{Variant 2}

For calculations below we defined restrictions as follows: kmin=10 and kmax=320. Which means that we were looking for $\mathrm{k}(\mathrm{z})$ distribution in this range: $10<\mathrm{k}(\mathrm{z})<320$. Numerical results are presented in table below.

\begin{tabular}{|c|c|c|c|c|c|c|}
\hline Simplex & Steps & $p_{i 0}$ & $p_{i 1}$ & $p_{i 2}$ & $p_{i 3}$ & Fmin \\
\hline A & 91 & $7.81939 \mathrm{e}+1$ & $2.99943 \mathrm{e}+2$ & $-6.54279 \mathrm{e}+1$ & 8.01293 & 2.21234 \\
\hline B & 156 & 9.98179 & 5.00957 & 9.61216 & $2.01388 \mathrm{e}+1$ & $3.02264 \mathrm{e}-1$ \\
\hline C & 117 & $3.98227 \mathrm{e}+1$ & $8.07309 \mathrm{e}+1$ & $-1.43192 \mathrm{e}+2$ & $2.37720 \mathrm{e}+2$ & $8.36632 \mathrm{e}-2$ \\
\hline
\end{tabular}

Table 12. Values of minimized parameters pmin for simplexes A, B, C

For the start simplex A, distribution of the thermal conductivity for minimized value $\mathrm{k}$ (pmin) is shown in Fig. 15. Disparity between the expected and the obtained temperature distribution was also examined (see Fig. 16) and it varies between -3.8 and 2.8.

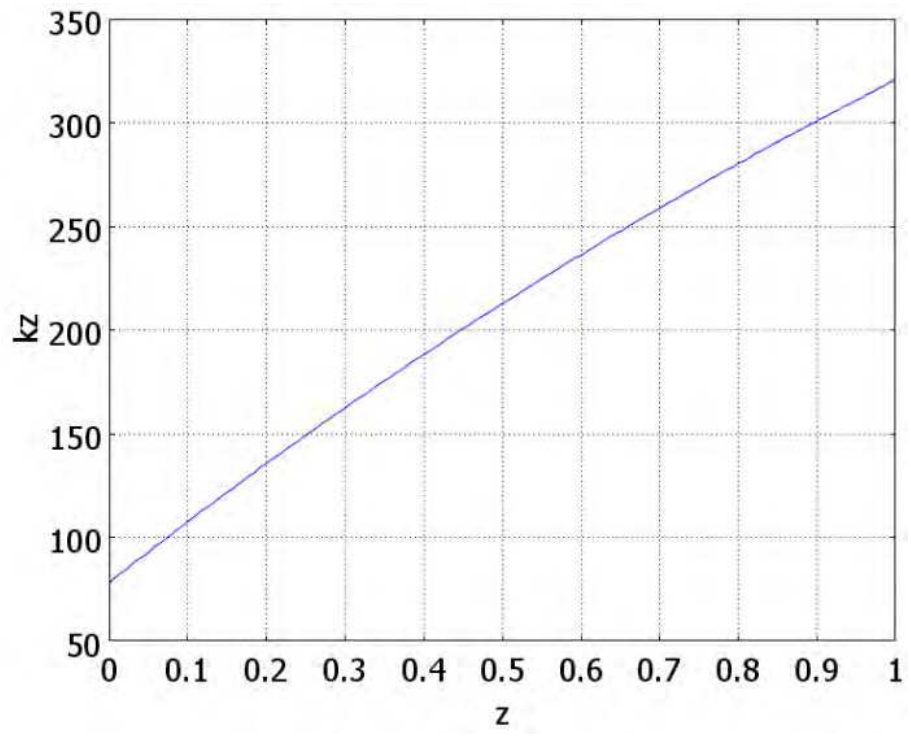

Fig. 15. Variant 2, start simplex A- Distribution of the thermal conductivity 


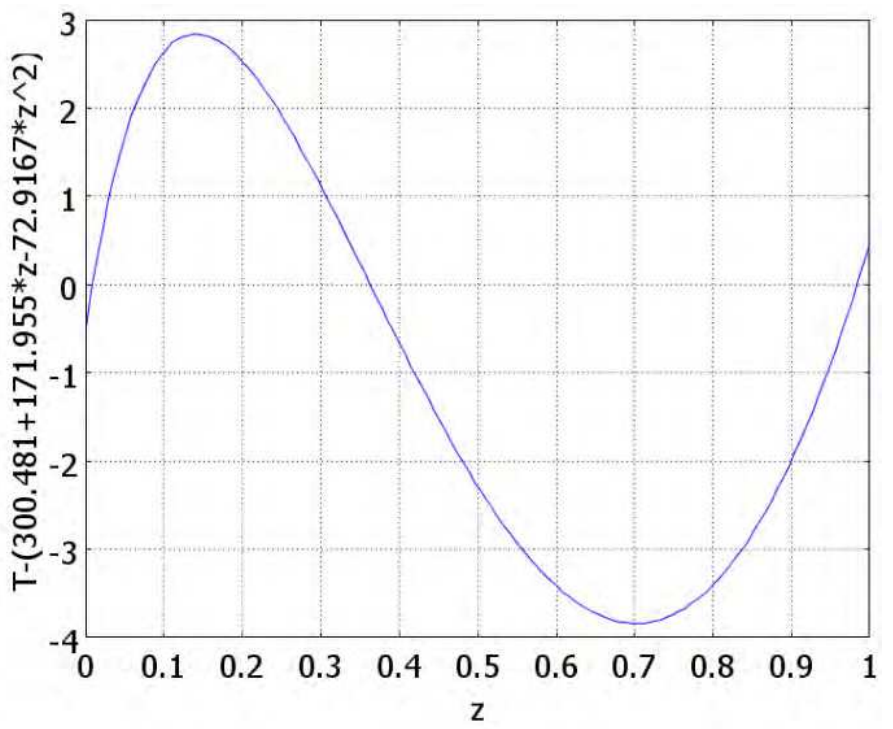

Fig. 16. Variant 2, start simplex A - Disparity between the expected and the obtained temperature distribution

For the start simplex $B$, distribution of the thermal conductivity for minimized value $\mathrm{k}(\mathrm{pmin})$ is shown in Fig. 17. Disparity between the expected and the obtained temperature distribution was also examined (see Fig. 18) and it varies between -0.56 and 0.46 .

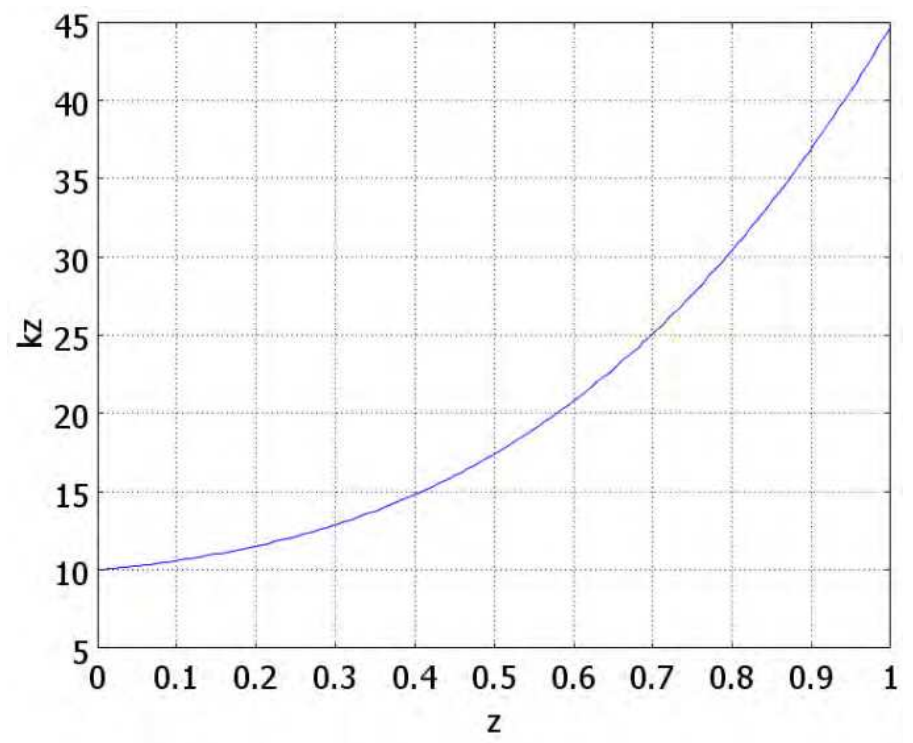

Fig. 17. Variant 2, start simplex B- Distribution of the thermal conductivity 


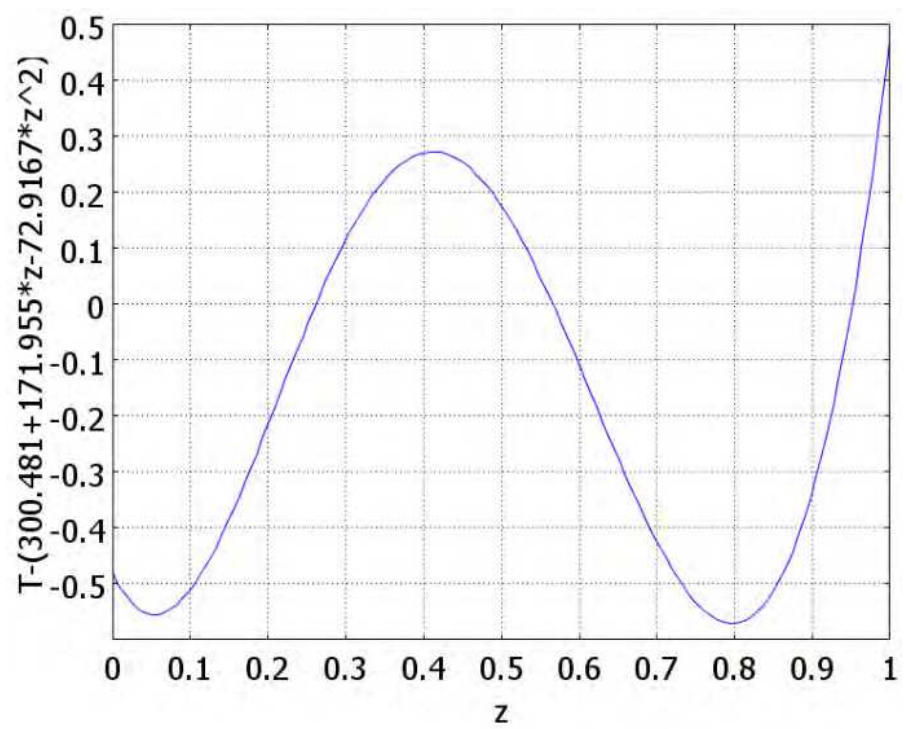

Fig. 18. Variant 2, start simplex B - Disparity between the expected and the obtained temperature distribution

For the start simplex $\mathrm{C}$, distribution of the thermal conductivity for minimized value $\mathrm{k}(\mathrm{pmin})$ is shown in Fig. 19. Disparity between the expected and obtained temperature distribution was also examined (see Fig. 20) and it varies between -0.48 and 0.47.

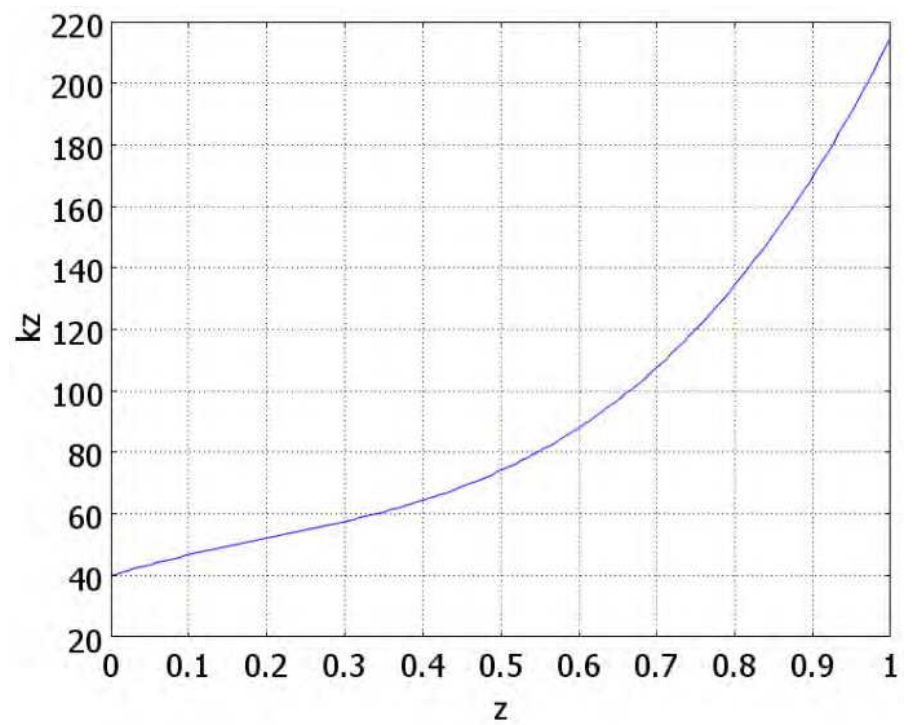

Fig. 19. Variant 2, start simplex C- Distribution of the thermal conductivity 


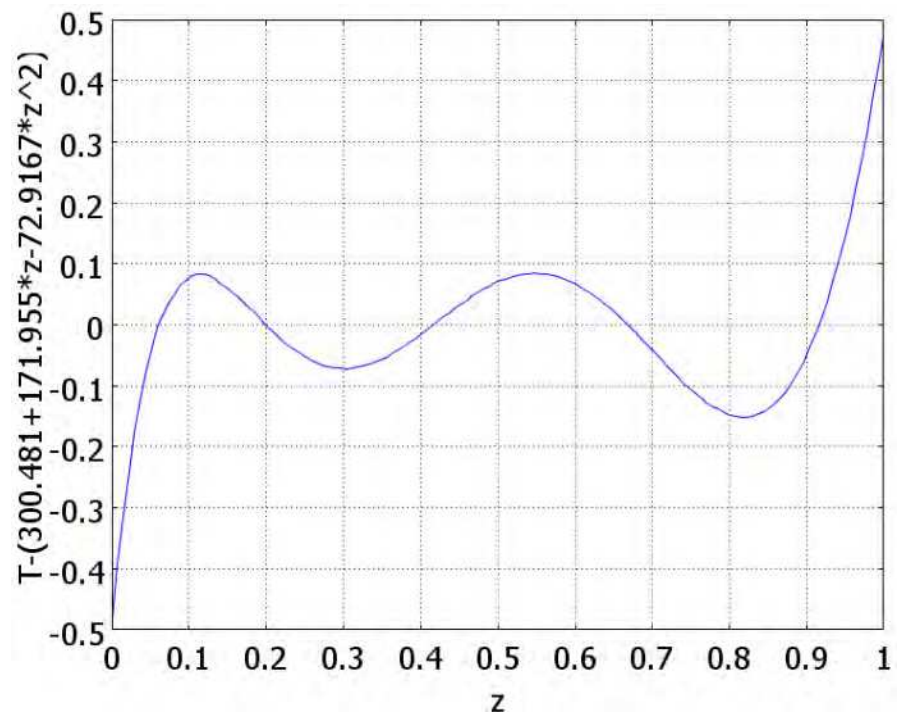

Fig. 20. Variant 2, start simplex C - Disparity between the expected and the obtained temperature distribution

In all cases the results were achieved with solution accuracy of 1e-5. Temperature distribution was similar for all simplexes.

\subsection{Task 2 - Second function of temperature distribution}

The form of the second expected temperature distribution looks as follows:

$$
\overline{\mathrm{T}}_{1}(\mathrm{z})=298.794+149.678 \cdot \mathrm{z}-47.9503 \cdot \mathrm{z}^{2},
$$

and integral I1 takes a form of

$$
\mathrm{I} 1=\int_{0}^{1} \operatorname{Abs}\left(\mathrm{T}(\mathrm{z})-\left(298.794+149.678 \cdot \mathrm{z}-47.9503 \cdot z^{2}\right)\right) \mathrm{dz} .
$$

For this task another three different start simplexes were assumed and collected in Table 13.

\begin{tabular}{|c|c|c|c|c|c|}
\hline & Vertices, $p_{i j}$ & $p_{i 0}$ & $p_{i 1}$ & $p_{i 2}$ & $p_{i 3}$ \\
\hline \multirow{4}{*}{$\begin{array}{c}\text { Start simplex A } \\
\text { (the narrowest) }\end{array}$} & $p_{1 j}$ & 10 & 150 & -80 & 0 \\
\cline { 2 - 6 } & $p_{2 j}$ & 15 & 160 & -90 & 1 \\
\cline { 2 - 6 } & $p_{3 j}$ & 30 & 210 & -110 & 1 \\
\cline { 2 - 6 } & $p_{4 j}$ & 40 & 220 & -120 & 0 \\
\hline \multirow{4}{*}{$\begin{array}{c}\text { Start simplex B } \\
\text { (wider) }\end{array}$} & $p_{5 j}$ & 50 & 230 & -130 & 1 \\
\cline { 2 - 6 } & $p_{1 j}$ & 100 & 400 & -90 & 20 \\
\cline { 2 - 6 } & $p_{2 j}$ & -60 & -300 & -230 & 40 \\
\cline { 2 - 6 } & $p_{3 j}$ & 90 & 350 & 60 & 200 \\
\cline { 2 - 6 } & $p_{4 j}$ & 40 & 220 & -120 & 0 \\
\hline
\end{tabular}




\begin{tabular}{|c|c|c|c|c|c|}
\hline & Vertices, $p_{i j}$ & $p_{i 0}$ & $p_{i 1}$ & $p_{i 2}$ & $p_{i 3}$ \\
\hline \multirow{4}{*}{$\begin{array}{c}\text { Start simplex C } \\
\text { (the widest) }\end{array}$} & $p_{1 j}$ & 150 & 150 & -95 & 30 \\
\cline { 2 - 6 } & $p_{2 j}$ & -80 & -350 & -290 & 50 \\
\cline { 2 - 6 } & $p_{3 j}$ & 100 & 380 & 70 & 220 \\
\cline { 2 - 6 } & $p_{4 j}$ & 50 & 280 & -180 & 20 \\
\hline & $p_{5 j}$ & 200 & 250 & -400 & 100 \\
\hline
\end{tabular}

Table 13. Task 2 - Different start simplexes taken for calculation

For this function also two variants were calculated. Results and assumptions for them are presented in chapters 5.2.1 and 5.2.2.

\subsubsection{Variant 1}

The restrictions defined for these variants take a form of: $k m i n=20$ and $k m a x=120$. This means that we were looking for $\mathrm{k}(\mathrm{z})$ distribution in range like: $20<\mathrm{k}(\mathrm{z})<120$. Numerical results are presented in table below.

\begin{tabular}{|c|c|c|c|c|c|c|}
\hline Simplex & Steps & $p_{i 0}$ & $p_{i 1}$ & $p_{i 2}$ & $p_{i 3}$ & Fmin \\
\hline A & 138 & $5.84429 \mathrm{e}+1$ & $-1.02247 \mathrm{e}+2$ & $3.68435 \mathrm{e}+2$ & $-2.21308 \mathrm{e}+2$ & $6.60791 \mathrm{e}-1$ \\
\hline B & 111 & $3.95147 \mathrm{e}+1$ & $5.94787 \mathrm{e}+1$ & $-1.13091 \mathrm{e}+2$ & $1.3497 \mathrm{e}+2$ & $3.07911 \mathrm{e}-1$ \\
\hline C & 240 & $4.55508 \mathrm{e}+1$ & $1.59731 \mathrm{e}+1$ & 7.83838 & $5.09419 \mathrm{e}+1$ & $2.02351 \mathrm{e}-1$ \\
\hline
\end{tabular}

Table 14. Values of minimized parameters pmin for simplexes A, B, C

For the start simplex A, distribution of the thermal conductivity for minimized value $\mathrm{k}$ (pmin) is plotted in Fig. 21. Between the expected and the obtained temperature distribution was some disparity which is from -1.48 and 1.2 (see Fig. 22).

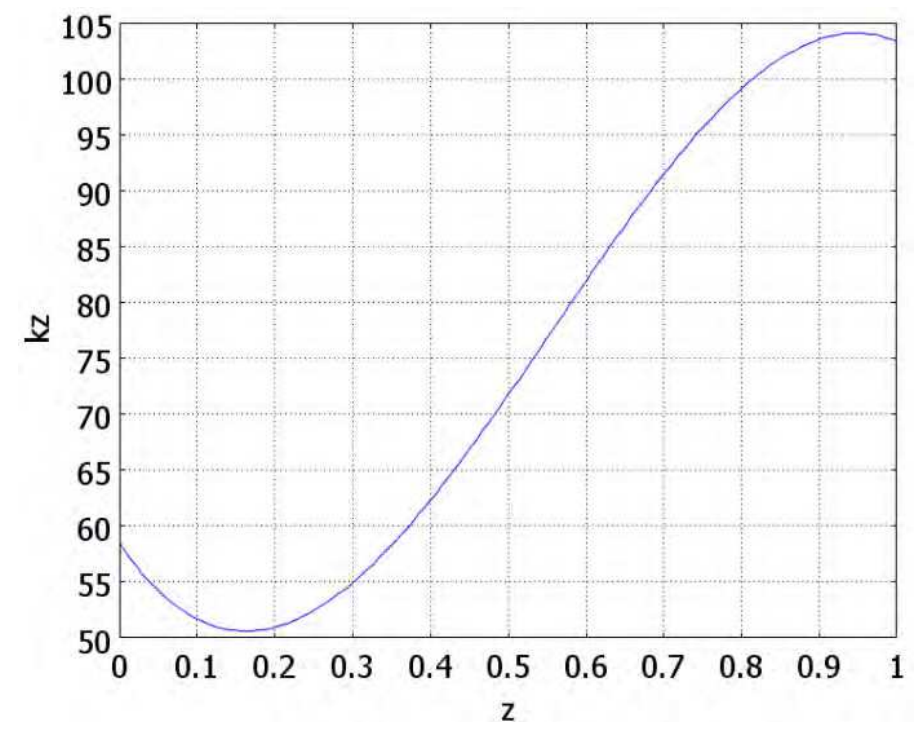

Fig. 21. Variant 1, start simplex A- Distribution of the thermal conductivity 


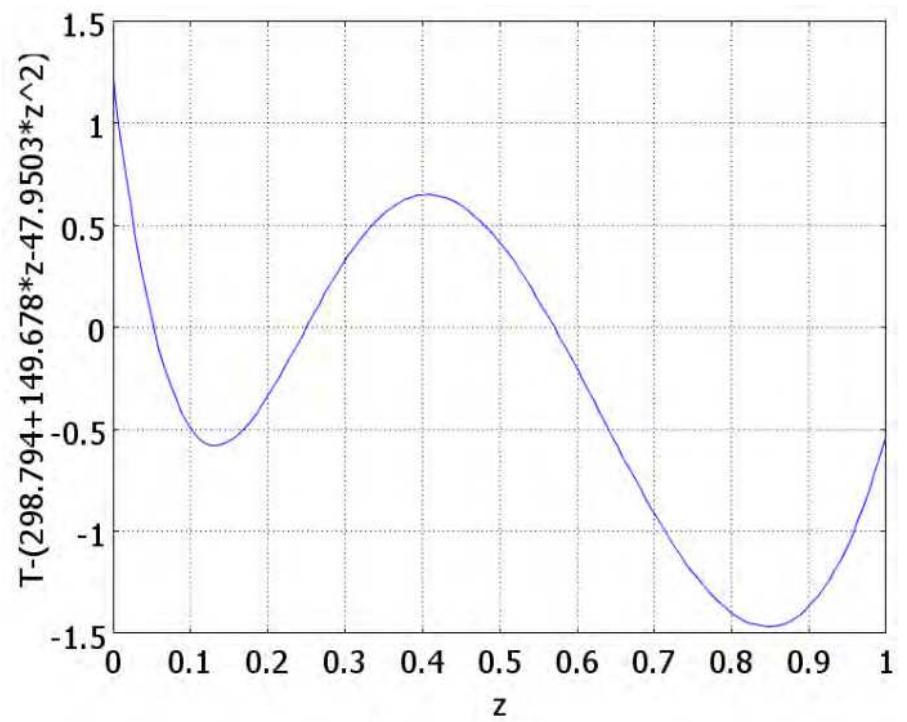

Fig. 22. Variant 1, start simplex A - Disparity between the expected and the obtained temperature distribution

For the start simplex B, distribution of thermal conductivity for minimized value $\mathrm{k}(\mathrm{pmin})$ is plotted in Fig. 23. It was some disparity, between the expected and the obtained temperature distribution, and value of it was from -0.45 to 1.21 (see Fig. 24).

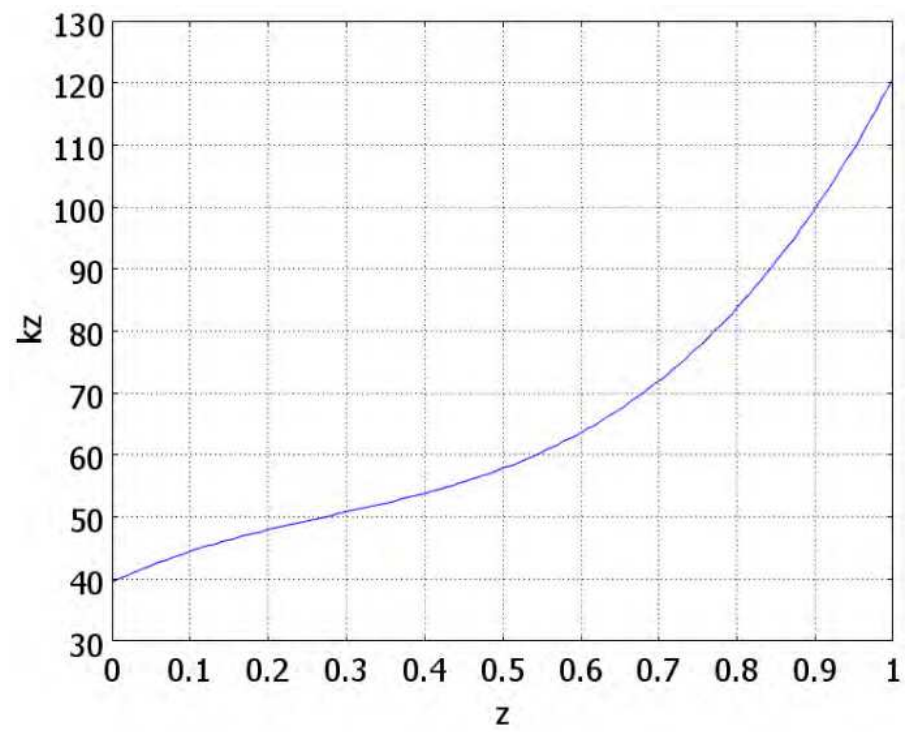

Fig. 23. Variant 1, start simplex B- Distribution of the thermal conductivity 


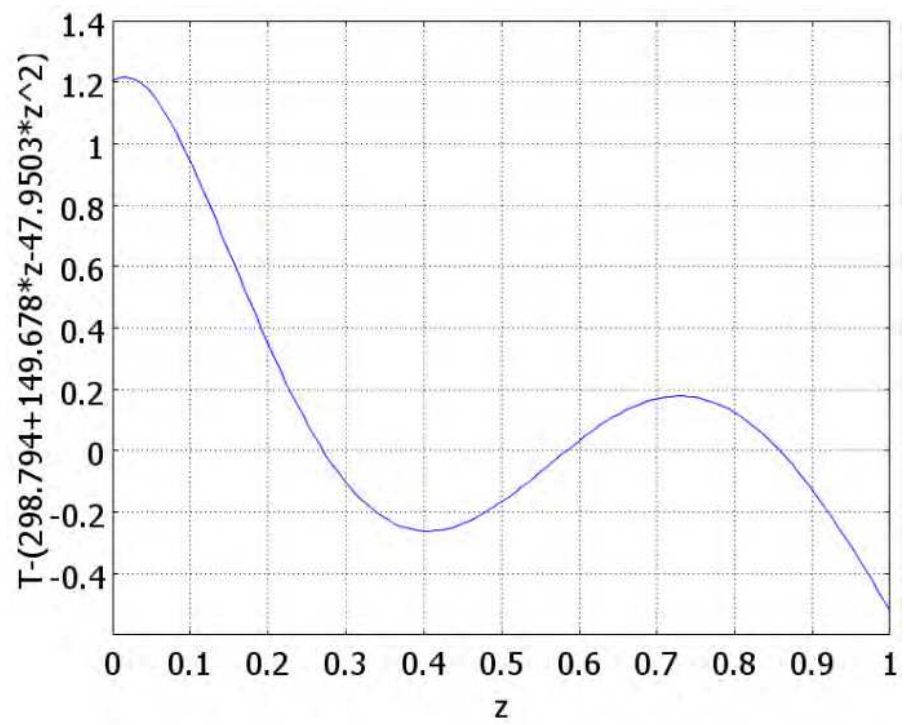

Fig. 24. Variant 1, start simplex B - Disparity between the expected and the obtained temperature distribution

For the start simplex $\mathrm{C}$, distribution of the thermal conductivity for minimized value $\mathrm{k}$ (pmin) is plotted in Fig. 25. It was some disparity, between the expected and the obtained temperature distribution, and value of it was from -0.45 to 1.2 (see Fig. 26).

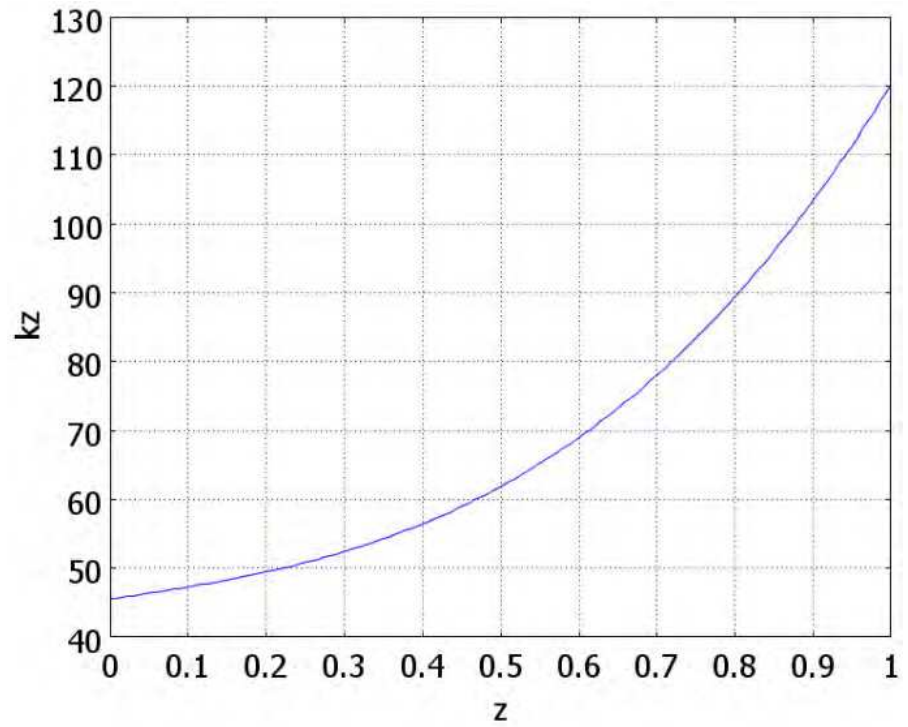

Fig. 25. Variant 1, start simplex C- Distribution of the thermal conductivity 


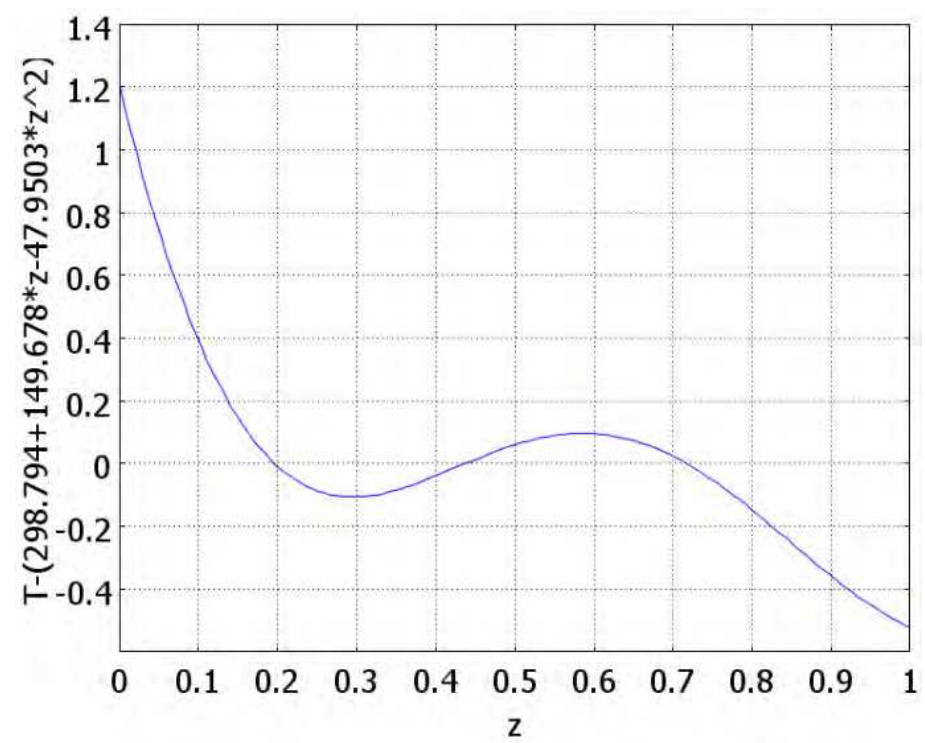

Fig. 26. Variant 1, start simplex C - Disparity between the expected and the obtained temperature distribution

\subsubsection{Variant 2}

The restrictions defined for these variants take a form of: $k m i n=10$ and $k m a x=320$. This means that we were looking for $\mathrm{k}(\mathrm{z})$ distribution in range like: $10<\mathrm{k}(\mathrm{z})<320$. Numerical results are presented in table below.

\begin{tabular}{|c|c|c|c|c|c|c|}
\hline Simplex & Steps & $p_{i 0}$ & $p_{i 1}$ & $p_{i 2}$ & $p_{i 3}$ & Fmin \\
\hline A & 129 & $1.34412 \mathrm{e}+2$ & $-1.60382 \mathrm{e}+2$ & $6.15741 \mathrm{e}+2$ & $-3.19053 \mathrm{e}+2$ & $4.65232 \mathrm{e}-1$ \\
\hline B & 146 & $1.13668 \mathrm{e}+2$ & $9.88941 \mathrm{e}+1$ & $-1.36084 \mathrm{e}+2$ & $2.45597 \mathrm{e}+2$ & $2.31297 \mathrm{e}-1$ \\
\hline C & 119 & $1.32751 \mathrm{e}+2$ & $-2.95152 \mathrm{e}+1$ & $2.27876 \mathrm{e}+2$ & -9.65976 & $2.4153 \mathrm{e}-1$ \\
\hline
\end{tabular}

Table 15. Values of minimized parameters pmin for simplexes A, B, C

In Fig. 27 the distribution of the thermal conductivity for minimized value $\mathrm{k}(\mathrm{pmin})$ is plotted for the start simplex A. It was some disparity, between the expected and the obtained temperature distribution, and value of it was from -1 to 1.2 (see Fig. 28). 


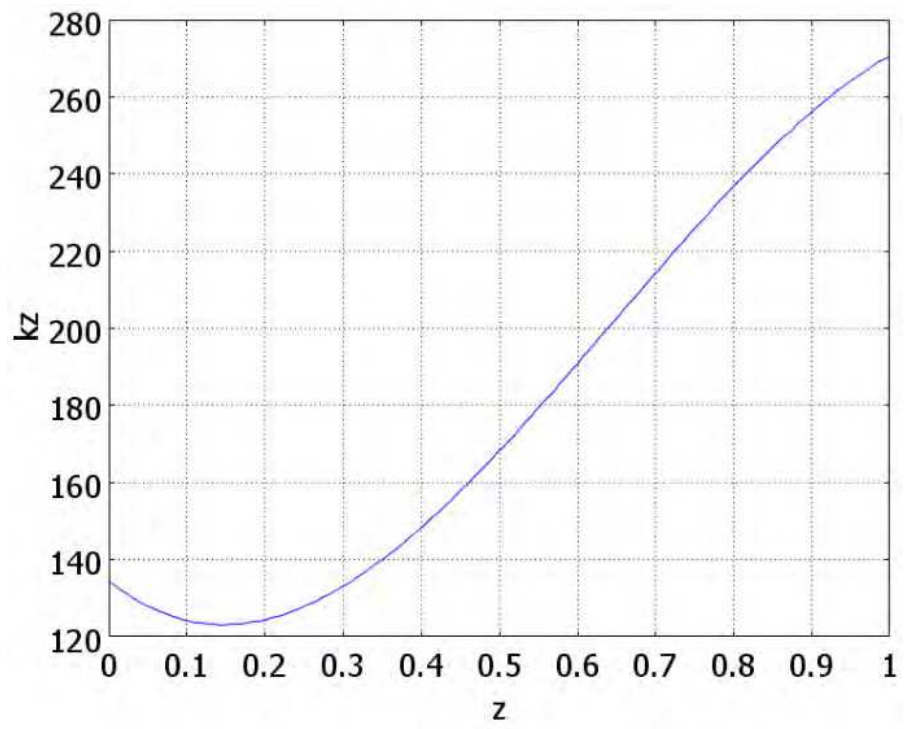

Fig. 27. Variant 2, start simplex A- Distribution of the thermal conductivity

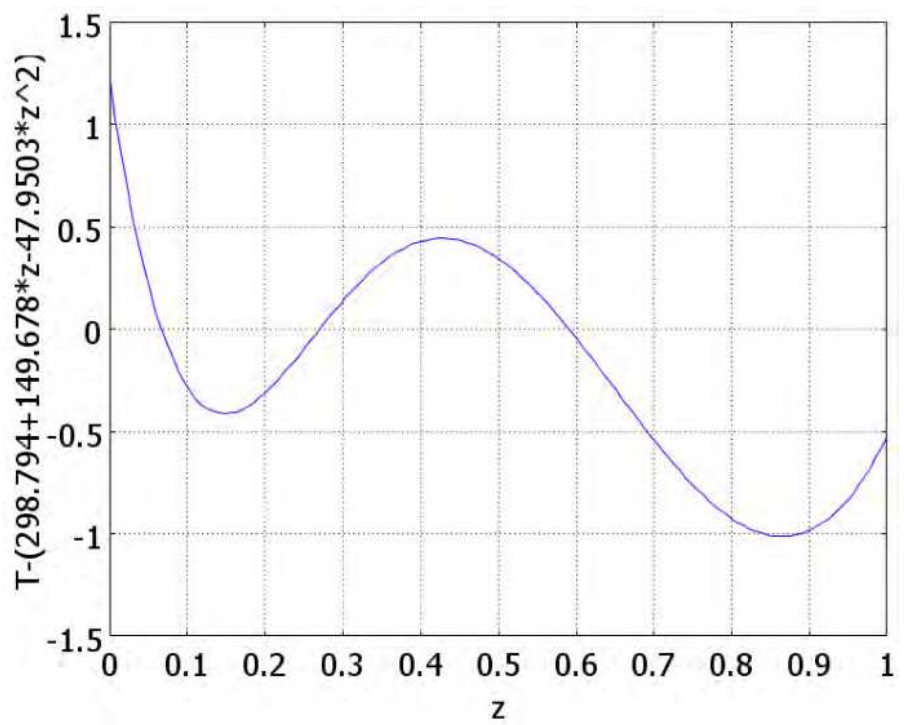

Fig. 28. Variant 2, start simplex A - Disparity between the expected and the obtained temperature distribution

In Fig. 29 distribution of the thermal conductivity for minimized value $\mathrm{k}(\mathrm{pmin})$ is plotted for the start simplex B. It was some disparity, between the expected and the obtained temperature distribution, and value of it was from -0.41 to 1.2 (see Fig. 30). 


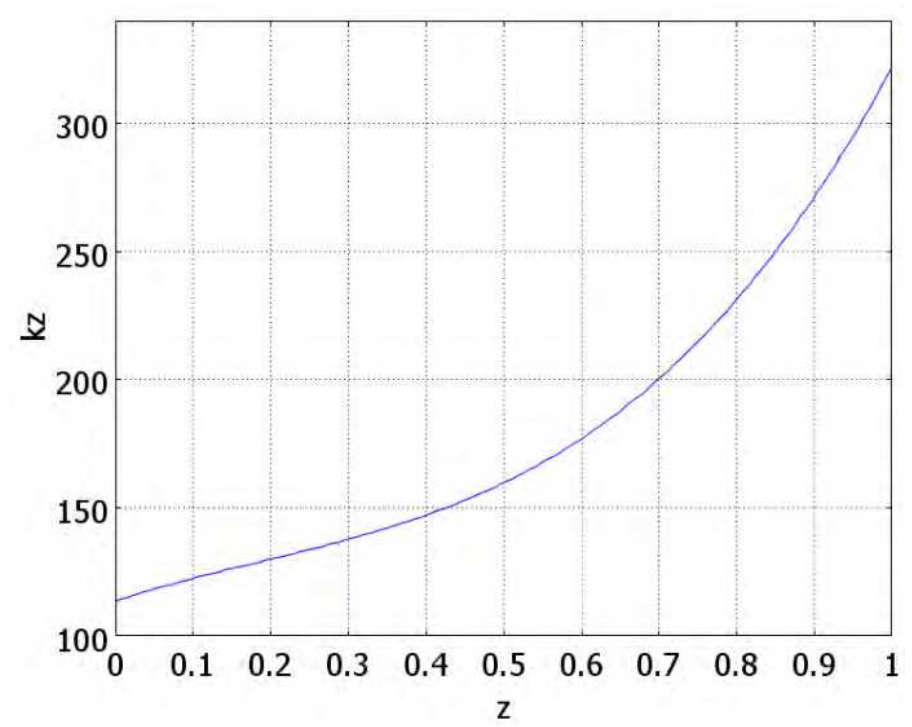

Fig. 29. Variant 2, start simplex B- Distribution of the thermal conductivity

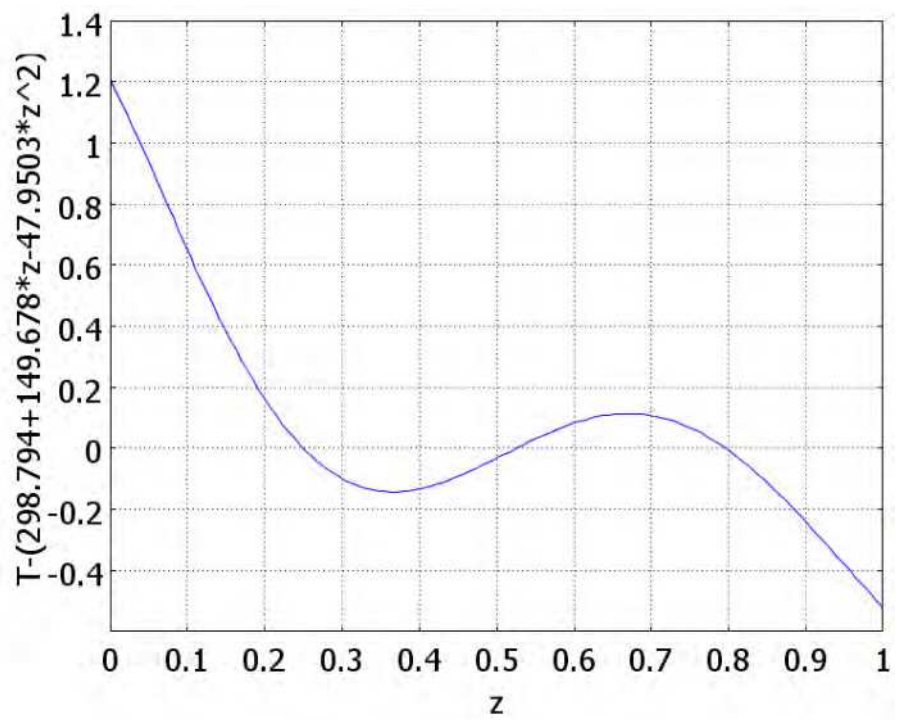

Fig. 30. Variant 2, start simplex B - Disparity between the expected and the obtained temperature distribution

In Fig. 31 the distribution of thermal conductivity for minimized value $\mathrm{k}$ (pmin) is plotted for the start simplex C. It was some disparity, between the expected and the obtained temperature distribution, and value of it was from -0.47 to 1.2 (see Fig. 32). 


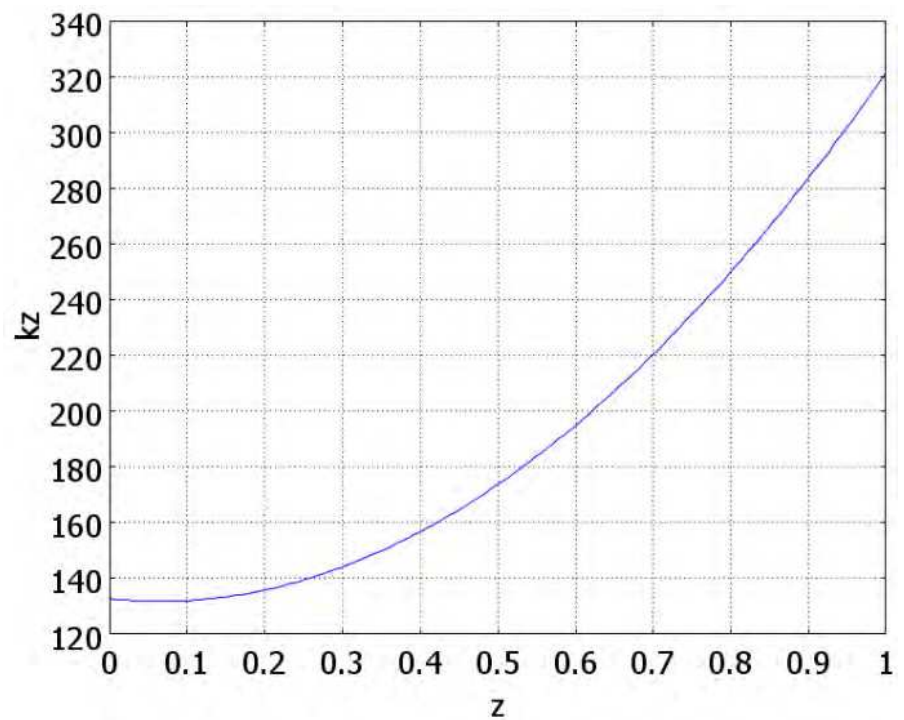

Fig. 31. Variant 2, start simplex C- Distribution of the thermal conductivity

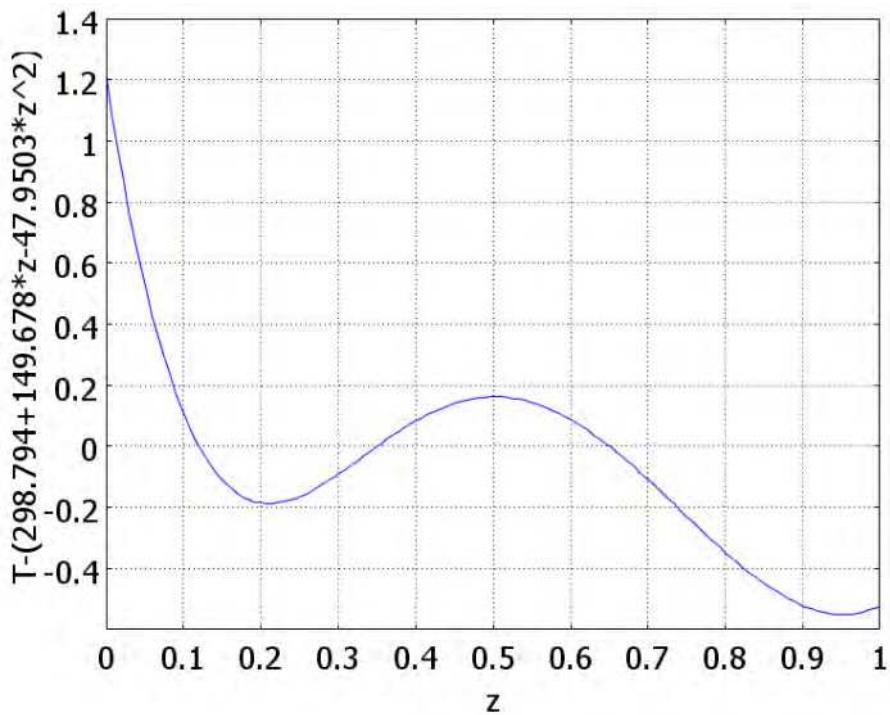

Fig. 32. Variant 2, start simplex C - Disparity between the expected and the obtained temperature distribution 
In all cases the results were achieved with solution accuracy of 1e-5. For all simplexes the temperature distribution was similar.

Summarizing, in this chapter some new possibilities of Identification of Thermal Conductivity of Modern Materials using the Finite Element Method and Nelder - Mead's Optimization Algorithm were proposed. Simulating heat transfer in 2-D axial symmetry model, made of the Functionally Graded Material, it is possible to designate its thermal conductivity distribution. This parameter can have the polynomial form. It is also possible to calculate its values within some restrictions. All solutions were found regardless of how far the start simplex was from the solution.

\section{Conclusion}

Because experimental evaluation of thermal parameters of composites is expensive and time consuming, computational methods have been found to be efficient alternatives for predicting the best parameters of composites. As it was presented in this chapter the NelderMead algorithm connected with the Finite Element Method can be used to optimize many different issues. It has its applicable in problems where it is difficult or impossible to designate the gradient of the objective function. The developed hybrid method can be used for optimization of the heat transfer problems.

In the section 4 of this chapter reconstruction of parameters was provided. Some heat transfer parameters in one-dimensional domain with length $1 \mathrm{~m}$, for defined boundary conditions were designated using numerical calculations. The thermal conductivity, the transversal convective heat transfer coefficient and the external temperature around both ends of the domain were calculated within some defined restrictions.

Next, in section 5 possibility of designation of the thermal conductivity was shown. The 2-D axial symmetry model was considered where heat transfer was simulated. The thermal conductivity was in polynomial form. There was also possible to put some restrictions on the searched parameters.

The hybrid method, which was proposed here, can be very helpful in designating any parameters of modern materials like for example Functionally Graded Materials. Proposed method can be also used instead of destructive testing of materials.

\section{References}

Bejan, A. \& Kraus, A. D. (2003). Heat transfer handbook, WILEY, ISBN 0-471-39015-1, USA.

Birman, V. \& Byrd, L. W. (2007). Modeling and Analysis of Functionally Graded Materials and Structures, Vol. 60, pp. 195-216. Available from http:/ / www.ewp.rpi.edu/hartford/ nelsob/EP/REFERENCES/Papers/ FGM\%20Modeling\%20and\%20Analysis.pdf

Blaheta, R., Kohut, R. \& Jakl, O. (2010). Solution of Identification Problems in Computational Mechanics - Parallel Processing Aspects, Available from http://vefir.hi.is/para10/extab/para10-paper-70.pdf. 
Borukhov, V. T. \& Timoshpol'skii, V. T. (2005). Functional Identification of the Nonlinear Thermal-Conductivity Coefficient by Gradient Methods. I. Conjugate Operators, Journal of Engineering Physics and Thermophysics, Vol. 78, No. 4, pp. 695-702.

Comsol Multiphysics User's Guide (2007). Modeling Guide and Model Library, Documentation Set, Comsol AB.

Durand, N. \& Alliot, J. M. (1999). A Combined Nelder-Mead Simplex and Genetic Algorithm, Available from http://recherche.enac.fr/opti/papers/articles/gecco99.pdf

Hossein Ghiasi, M., Pasini, D. \& Lessard, L. (2007). Improved Globalized Nelder-Mead Method for Optimization of a Composite Bracket, Available from http://www.iccmcentral.org/Proceedings/ICCM16proceedings/contents/pdf/ MonK/MoKA1-04ge_ghiasimh224461p.pdf

Jingchuan, Z., Zhongda, Y., Zhonghong, L. \& Jian, L. (1996). Microstructure and thermal stress relaxation of ZrO2-Ni Functionally Graded Material, Vol. 6, No. 4, pp. 94-99.

Jopek, H. \& Strek, T. (2011). Optimization of the Effective Thermal Conductivity of a Composite, Convection and Conduction Heat Transfer, Amimul Ahsan (Ed.), InTech, ISBN 978-953-307-582-2. Available from

http://www.intechopen.com/articles/show/title/optimization-of-the-effectivethermal-conductivity-of-a-composite

Lasheen, A. A., El-Garhy, A. M., Saad, E. M. \& Eid, S. M. (2009). Using hybrid genetic and Nelder-Mead algorithm for decoupling of MIMO system with application on two coupled distillation columns process, Available from

http://www.naun.org/journals/mcs/mcs-124.pdf

Luersen, M. A. \& Le Riche, R. (2004). Globalized Nelder-Mead method for engineering optimization, Available from http://www.sciencedirect.com/science/article/pii/S0045794904002378

Mastorakis, N. E. (2009). Genetic Algorithms with Nelder-Mead Optimization in the variational methods of Boundary Value Problems, Vol. 8, No. 3, pp. 107-116, ISSN: 1109-2769

Miyamoto, Y., Kaysser, W. A., Rabin, B. H., Kawasaki, A. \& Ford, R. G. (1999). Functionally Graded Materials: Design, Processing and Applications, Kluwer Academic Publishers, ISBN 0-412-60760-3, USA

Nansen, N. (2009). Benchmarking the Nelder-Mead Downhill Simplex Algorithm With Many Local Restarts, Available from http://hal.archives-ouvertes.fr/inria-00382104/en

Nelder, J. A. \& Mead, R. (1965). Simplex method for function minimization, Computer Journal, Vol. 7. No. 4, pp 308-313

Stefanescu, S. (2007). Applying Nelder Mead's Optimization Algorithm for Multiple Global Minima, Romanian Journal for Economic Forecasting. Available from http://www.ipe.ro

Weise, T. (2009). Global Optimization Algorithms- Theory and Application, Available from http://www.it-weise.de/ 
Zienkiewicz, O.C. \& Taylor, R.L. (2000). The Finite Element Method, Vol. 1-3: The Basis, Solid Mechanics, Fluid Dynamics (5th ed.), Butterworth-Heinemann, Oxford. 


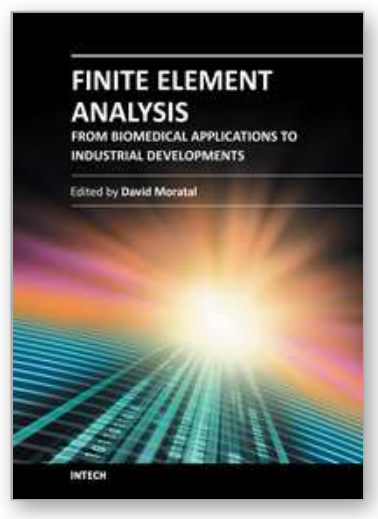

\author{
Finite Element Analysis - From Biomedical Applications to \\ Industrial Developments \\ Edited by Dr. David Moratal
}

ISBN 978-953-51-0474-2

Hard cover, 496 pages

Publisher InTech

Published online 30, March, 2012

Published in print edition March, 2012

Finite Element Analysis represents a numerical technique for finding approximate solutions to partial differential equations as well as integral equations, permitting the numerical analysis of complex structures based on their material properties. This book presents 20 different chapters in the application of Finite Elements, ranging from Biomedical Engineering to Manufacturing Industry and Industrial Developments. It has been written at a level suitable for use in a graduate course on applications of finite element modelling and analysis (mechanical, civil and biomedical engineering studies, for instance), without excluding its use by researchers or professional engineers interested in the field, seeking to gain a deeper understanding concerning Finite Element Analysis.

\title{
How to reference
}

In order to correctly reference this scholarly work, feel free to copy and paste the following:

Maria Nienartowicz and Tomasz Strek (2012). Identification of Thermal Conductivity of Modern Materials Using the Finite Element Method and Nelder-Mead's Optimization Algorithm, Finite Element Analysis - From Biomedical Applications to Industrial Developments, Dr. David Moratal (Ed.), ISBN: 978-953-51-0474-2, InTech, Available from: http://www.intechopen.com/books/finite-element-analysis-from-biomedicalapplications-to-industrial-developments/identification-of-thermal-conductivity-of-modern-materials-using-thefinite-element-method-and-nelde

\section{INTECH}

open science | open minds

\section{InTech Europe}

University Campus STeP Ri

Slavka Krautzeka 83/A

51000 Rijeka, Croatia

Phone: +385 (51) 770447

Fax: +385 (51) 686166

www.intechopen.com

\section{InTech China}

Unit 405, Office Block, Hotel Equatorial Shanghai

No.65, Yan An Road (West), Shanghai, 200040, China

中国上海市延安西路65号上海国际贵都大饭店办公楼 405 单元

Phone: +86-21-62489820

Fax: $+86-21-62489821$ 
(C) 2012 The Author(s). Licensee IntechOpen. This is an open access article distributed under the terms of the Creative Commons Attribution 3.0 License, which permits unrestricted use, distribution, and reproduction in any medium, provided the original work is properly cited. 